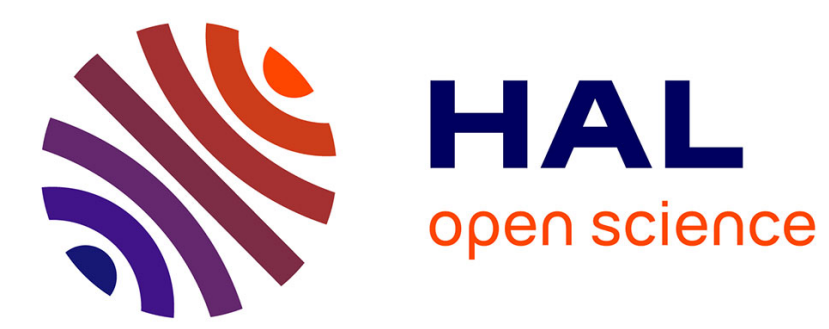

\title{
The first cycles in an evolving graph
}

\author{
Philippe Flajolet, Boris Pittel, Donald E. Knuth
}

\section{To cite this version:}

Philippe Flajolet, Boris Pittel, Donald E. Knuth. The first cycles in an evolving graph. [Research

Report] RR-0888, INRIA. 1988. inria-00075666

\section{HAL Id: inria-00075666 \\ https://hal.inria.fr/inria-00075666}

Submitted on 24 May 2006

HAL is a multi-disciplinary open access archive for the deposit and dissemination of scientific research documents, whether they are published or not. The documents may come from teaching and research institutions in France or abroad, or from public or private research centers.
L'archive ouverte pluridisciplinaire HAL, est destinée au dépôt et à la diffusion de documents scientifiques de niveau recherche, publiés ou non, émanant des établissements d'enseignement et de recherche français ou étrangers, des laboratoires publics ou privés. 


\title{
Rapports de Recherche
}

\section{$\mathbf{N}^{\circ} 888$}

\section{THE FIRST CYCLES IN AN EVOLVING GRAPH}

\author{
Philippe FLAJOLET \\ Donald E. KNUTH \\ Boris PITTEL
}

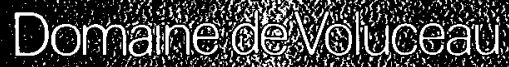

Ror. ถาำ.

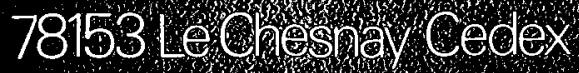
1.

Tél. 


\title{
The First Cycles in an Evolving Graph
}

\author{
Philippe Flajolet, Donald E. Knuth and Boris Pittel
}

\begin{abstract}
If successive connections are added at random to an initially disconnected set of $n$ points, the expected length of the first cycle that appears will be proportional to $n^{1 / 6}$, with a standard deviation proportional to $n^{1 / 4}$. The size of the component containing this cycle will be of order $n^{1 / 2}$, on the average, with standard deviation of order $n^{7 / 12}$. The average length of the $k$ th cycle is proportional to $n^{1 / 6}(\log n)^{k-1}$. Furthermore, the probability is $\sqrt{2 / 3}+O\left(n^{-1 / 3}\right)$ that the graph has no components with more than one cycle at the moment when the number of edges passes $\frac{1}{2} n$. These results can be proved with analytical methods based on combinatorial enumeration with multivariate generating functions, followed by contour integration to derive asymptotic formulas for the quantities of interest.
\end{abstract}

\section{Les premiers cycles dans un graphe en évolution}

\author{
Philippe Flajolet, Donald E. Knuth et Boris Pittel
}

Résumé. Si l'on ajoute aléatoirement des arêtes à un ensemble de $n$ points initiallement disconnectés, la longueur moyenne du premier cycle qui apparaît est proportionnelle à $n^{1 / 6}$ avec un écart type proportionnel à $n^{1 / 4}$. La taille du composant contenant ce cycle est de l'ordre de $n^{1 / 2}$ en moyenne, et l'écart type de l'ordre de $n^{7 / 12}$. La longueur moyenne du $k$-ième cycle est proportionnelle à $n^{1 / 6}(\log n)^{k-1}$. De surcroît, lorsque le nombre d'arêtes atteint la valeur $n / 2$, il y a une probabilité $\sqrt{2 / 3}+O\left(n^{-1 / 3}\right)$ que le graphe ne contienne pas de composante connexe ayant plus d'un cycle. Ces résultats sont établis par des méthodes analytiques fondées sur l'utilisation de séries génératrices de dénombrement multivariées, suivies d'intégration le long de contours appropriés. L'on obtient de la sorte les estimations asymptotiques de divers paramètres caractéristiques du processus d'évolution.

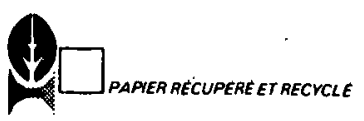




\title{
The First Cycles in an Evolving Graph
}

\author{
by Philippe Flajolet, ${ }^{1}$ Donald E. Knuth, ${ }^{2}$ and Boris Pittel ${ }^{3}$ \\ Computer Science Department, Stanford University
}

\section{Dedicated to Paul Erdős on his 75th birthday}

\begin{abstract}
If successive connections are added at random to an initially disconnected set of $n$ points, the expected length of the first cycle that appears will be proportional to $n^{1 / 6}$, with a standard deviation proportional to $n^{1 / 4}$. The size of the component containing this cycle will be of order $n^{1 / 2}$, on the average, with standard deviation of order $n^{7 / 12}$. The average length of the $k$ th cycle is proportional to $n^{1 / 6}(\log n)^{k-1}$. Furthermore, the probability is $\sqrt{2 / 3}+O\left(n^{-1 / 3}\right)$ that the graph has no components with more than one cycle at the moment when the number of edges passes $\frac{1}{2} n$. These results can be proved with analytical methods based on combinatorial enumeration with multivariate generating functions, followed by contour integration to derive asymptotic formulas for the quantities of interest.
\end{abstract}

1980 Mathematics Subject Classification (1985 Revision)

Primary Numbers: 05C80 (Random graphs), $05 \mathrm{A15}$ (Combinatorial enumeration)

Secondary Numbers: 41A60 (Asymptotic approximations), 68Q25 (Analysis of algorithms)

1 Permanent address: INRIA, Rocquencourt, 78150 Le Chesnay (France).

2 Permanent address: Computer Science Department, Stanford University, Stanford, CA 94305 (USA).

3 Permanent address: Mathematics Department, Ohio State University, Columbus, $O H$ 43210 (USA).

This research was supported in part by the National Science Foundation under grant CCR-86-10181, and by Office of Naval Research contract N00014-87-K-0502. 
A classic paper by Erdös and Renyi [ER59] inaugurated the study of the random graph process, in which we begin with a totally disconnected graph and enrich it by successively adding edges. Algorithms that deal with graphs often mimic such a process, inputting a sequence of edges until some stopping criterion occurs, based on the configuration of edges seen so far. To analyze such algorithms, we wish to estimate relevant characteristics of the resulting graph. For example, we might stop when the graph first contains a particular kind of subgraph, and we might ask how large that subgraph is.

The purpose of this paper is to introduce analytical methods by which such questions can be answered systematically. In particular, we will apply the ideas to an interesting question posed by Paul Erdős and communicated by Edgar Palmer to the 1985 Seminar on Random Graphs in Posnan: "What is the expected length of the first cycle in an evolving graph?" The answer turns out to be rather surprising: The first cycle has length $K n^{1 / 6}+O\left(n^{1 / 8}\right)$ on the average, where

$$
K=\frac{1}{\sqrt{8 \pi} i} \int_{-\infty}^{\infty} \int_{1-i \infty}^{1+i \infty} e^{(\mu+2 s)(\mu-s)^{2} / 6} \frac{d s}{s} d \mu \approx 2.0337
$$

The form of this result suggests that the expected behavior may be quite difficult to derive using techniques that do not use contour integration.

The methods to be described start with comparatively easy techniques of combinatorial analysis based on generating functions, and finish with more difficult (yet standard) techniques of complex analysis. The main novelty in this approach is the use of contour integration to give parametric estimates of a function that appears within an ordinary integral. Such methods may well find application in other studies of random graphs, so they are presented here in an expository fashion and in somewhat greater generality than is needed to solve the special problems used as examples.

Section 1 introduces two basic models of evolving graphs that will be studied in the sequel, corresponding roughly to sampling with and without replacement. Section 2 discusses bivariate generating functions suitable for studying these graphs. Such generating functions can be used to derive probabilities in both of the models, as shown in Section 3 . Asymptotic calculations in Section 4, based on the saddle point method, lead to results in Section 5 about the limiting distribution of first cycle lengths. Section 6 proves the main theorem about expected cycle length, and Section 7 derives auxiliary results about the expected waiting time and expected component sizes. The joint distribution of cycle lengths and edges is studied in Section 8, which also demonstrates a connection between waiting times and the parametric functions of Section 3. Section 9 extends the ideas to another problem in which we consider the first "bicyclic" component instead of the first cycle. An alternative approach to waiting times is considered in Section 10, where we also give an affirmative answer to a long-standing conjecture of Erdős and Renyi about the probability that a graph is planar. Finally we consider the first $k$ cycles, in Section 11. 
1. Models of graph evolution. We shall consider two related ways to enrich an initially empty graph on the vertices $\{1,2, \ldots, n\}$. The first procedure, called the uniform model, is the simplest: At each step we generate an ordered pair $\langle x, y\rangle$, where $x$ and $y$ are uniformly distributed between 1 and $n$, and all $n^{2}$ pairs are equally likely. The (undirected) edge $x-y$ is then added to the graph. In this way we obtain a multigraph, which may have duplicate edges or self-loops $x-x$. Interesting variants of this model can be obtained by imposing other distributions on the pairs $\langle x, y\rangle$, but we shall not pursue such generalizations in the present paper.

Another way to generate a sequence of random edges may be called the permutation model; this model corresponds directly to random graphs as studied in the classic papers by Erdős and Rényi [ER59, ER60]. Here we consider all $\left(\begin{array}{l}n \\ 2\end{array}\right) !$ permutations of the pairs $\langle x, y\rangle$ with $1 \leq x<y \leq n$ to be equally likely, and we generate new edges $x-y$ by considering the pairs as they occur in such a permutation. The resulting graph contains no self-loops and no multiple edges; we are essentially sampling without replacement.

The permutation model can be derived from the uniform model if we generate $\langle x, y\rangle$ uniformly but disregard any pairs with $x=y$ or pairs that duplicate a previous edge.

Our goal is to study the generation of random edges in such models until a cycle first appears in the resulting graph. (This would be the first time that a sequence of random "union" operations specifies a redundant union; see [KS78].) In the uniform model, the process might stop with a self-loop $\langle x, x\rangle$, which is a cycle of length 1 . Or it might stop with a duplicate edge (a pair $\langle x, y\rangle$ such that either $\langle x, y\rangle$ or $\langle y, x\rangle$ has occurred before); this is a cycle of length 2 . In the permutation model all cycles have length 3 or more.

For example, Figure 1 illustrates a "random" graph on $n=100$ vertices, based on the representation of $\pi=3.1415926 \ldots$ in decimal notation. (Here the vertices have been labeled 00 to 99 instead of 1 to $n$.) A cycle first appears when the 45th random pair, $\langle 05,55\rangle$, is added. In this case the uniform and permutation models produce identical graphs, because the first cycle has length $>2$; in other words, no duplicate edges or selfloops are generated before there is a cycle. (We will see in Theorem 2 below that both models give the same graph with probability approaching 8/15.)

The permutation model of graph evolution is often called the "random graph process." In these terms we can call the uniform graph model the "random multigraph process."

Let us recall briefly some of the main results of Erdős and Renyi from [ER60], to establish a context for the facts proved below. (A detailed discussion of the theory appears in [Bo85] and [Pa85].) The following properties hold "almost surely" (i.e., with probability tending to 1 as $n \rightarrow \infty)$ at the time when $m$ random edges have been added to an initially disconnected set of $n$ vertices: Only isolated vertices and edges will be present when $m \ll n^{1 / 2}$; but trees of order 3 will start to appear at time $m \approx n^{1 / 2}$, and trees of order 4 at time $m \approx n^{2 / 3}, \ldots$, trees of order $k+1$ at time $m \approx n^{1-1 / k}$. 

(00) (11) (03) (04) (07)
(10) (14)
(15)
(18)
(22)
(24) (29) (32) (36) (39)
(43) (44) (47)
(49) (54)
(56) (57)
6061
(63) 6
(68) 69
(71) (72) 73
$\begin{array}{lllll}755 & 76 & 77 & 79 & 85\end{array}$
(87) (90 96
(27)-95
(59)
(33)-83
(31) (46)

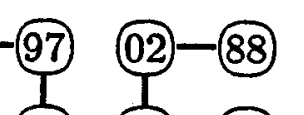

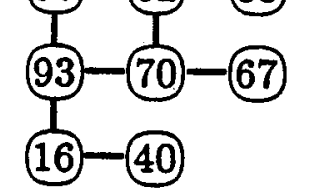
$\frac{(30)}{78}$
(99)-37
(45)

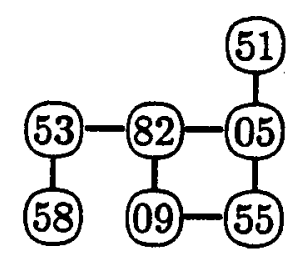

Figure 1. The final state of a graph on 100 vertices that has evolved until a cycle first appears. The successive ordered pairs $\langle 31,41\rangle\langle 59,26\rangle\langle 53,58\rangle\langle 97,93\rangle\langle 23,84\rangle\langle 62,64\rangle$ $\langle 33,83\rangle\langle 27,95\rangle\langle 02,88\rangle\langle 41,97\rangle\langle 16,93\rangle\langle 99,37\rangle\langle 51,05\rangle\langle 82,09\rangle\langle 74,94\rangle\langle 45,92\rangle\langle 30,78\rangle$ $\langle 16,40\rangle\langle 62,86\rangle\langle 20,89\rangle\langle 98,62\rangle\langle 80,34\rangle\langle 82,53\rangle\langle 42,11\rangle\langle 70,67\rangle\langle 98,21\rangle\langle 48,08\rangle\langle 65,13\rangle$ $\langle 28,23\rangle\langle 06,64\rangle\langle 70,93\rangle\langle 84,46\rangle\langle 09,55\rangle\langle 05,82\rangle\langle 23,17\rangle\langle 25,35\rangle\langle 94,08\rangle\langle 12,84\rangle\langle 81,11\rangle$ $\langle 74,50\rangle\langle 28,41\rangle\langle 02,70\rangle\langle 19,38\rangle\langle 52,11\rangle$ produce nothing but free trees in the initially empty graph, but then $\langle 05,55\rangle$ yields a cycle of length 4 . (This cycle is in the lower right corner.) At this point there still are 39 isolated vertices (shown at the upper left) that have not yet been mentioned.

There is (almost surely) no cycle while $m \ll n$. Later, when $m=\lambda n / 2$ and $\lambda<1$, there is at most one cycle in each component, and the largest component almost surely has size $\Theta(\log n)$. A dramatic phase transition occurs near $m=n / 2$, when one or several large components of size about $n^{2 / 3}$ appear. Still later, when $m=\lambda n / 2$ and $\lambda>1$, we find a single "giant" component of size $\Theta(n)$.

We wish to examine the state of the graph when the first cycle appears. According to [ER60], this almost surely happens at some time $m<n / 2$; we will see (Section 7 , 
Corollary 3) that the expected time is $m \approx n / 3$ in the uniform model, $m \approx 0.44 n$ in the permutation model. There still remain $\Theta(n)$ isolated vertices when the first cycle is formed (Corollary 4). And at this time the expected cycle length is of order $n^{1 / 6}$ (Theorem 3 ), with standard deviation of order $n^{1 / 4}$ (Section 7). The size of the component containing the first cycle will be $\Theta\left(n^{1 / 2}\right)$, with standard deviation of order $n^{7 / 12}$ (Corollary 1). We can also characterize the limit distribution of the first cycle length (Section 5, Theorem 2), as well as the limit distribution of the first cyclic component size (Section 7, Corollary 2). These distributions have a very slowly decaying tail and an infinite mean; hence their expected values of order $n^{1 / 6}$ and $n^{1 / 2}$ do not contradict the fact that the largest component almost surely has size $O(\log n)$ when $m / n, \leq \frac{1}{2}-\delta$.

With the same methods we will also gain some insight into events that take place around the time $m \approx n / 2$. The first bicyclic component (Section 9) appears at time $n / 2+\Theta\left(n^{2 / 3}\right)$, and its size is then of order $n^{2 / 3}$ (Corollary 5). However, at time $m=n / 2$ and a little beyond, there still is a positive probability that the graph will have no bicyclic component (Theorem 5 and Corollary 7); it will therefore still be planar.

2. Generating functions for stopping conflgurations. Probabilities and expected values in such random models can be obtained from generating functions whose coefficients count the number of graphs with specified characteristics and specified weights.

In our case we wish to count graphs that have a single cycle. Such graphs can conveniently be regarded as an unordered set of unrooted trees (representing the acyclic components) together with an ordered sequence of rooted trees (representing the component that has a cycle). For example, the graph of Figure 1 contains $\mathbf{4 0}$ isolated vertices, 11 vertex pairs that are (unrooted) trees of size 2 , and additional trees of respective sizes 4,5 , 6 , and 16; these are the acyclic components. The cyclic component is represented by a sequence of $l$ rooted trees, where $l$ is the length of the cycle, and the roots are the vertices of the cycle. In Figure 1, this sequence is

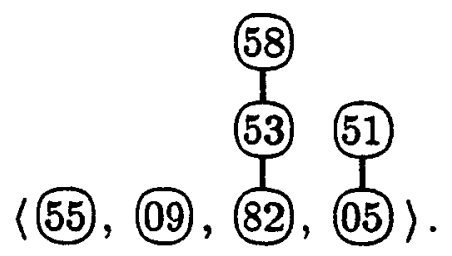

If the final cycle-completing edge in the random model was $\langle x, y\rangle$, we arrange the sequence of rooted trees so that the first root is $y$ and the last root is $x$. We shall say that a collection of unrooted and rooted trees as just described is a stopping configuration.

The enumeration of such labeled objects with exponential generating functions is a standard exercise in combinatorial analysis (see, for example, [CO70], [HP73], or [GJ83]), but it will be helpful to review the basic ideas briefly. If $F(z)$ is a power series, we write $\left[z^{n}\right] F(z)$ for the coefficient of $z^{n}$. We say that $F(z)$ is the exponential generating function 
(egf) for a collection $F$ of labelled objects if $n !\left[z^{n}\right] F(z)$ is the number $f_{n}$ of ways to attach labels to objects in $F$ that have $n$ elements, i.e., if

$$
F(z)=\sum_{n \geq 0} f_{n} \frac{z^{n}}{n !}=\sum_{A \in F} \frac{z^{\|A\|}}{\|A\| !} .
$$

If $F_{1}(z), \ldots, F_{k}(z)$ are egfs for $F_{1}, \ldots, F_{k}$, respectively, then the product $F_{1}(z) \ldots F_{k}(z)$ is the egf for all ordered sequences $\left\langle A_{1}, \ldots, A_{k}\right\rangle$ where $A_{j}$ is an element of $F_{j}$ with an appropriate relabeling. In particular, if $F_{1}(z)=\cdots=F_{k}(z)=F(z)$, then the functions

$$
F(z)^{k} \quad \text { and } \quad F(z)^{k} / k !
$$

exponentially generate sequences and sets, respectively, of $k$ objects from $f$. Summing over $k$, the functions

$$
\frac{1}{1-F(z)} \quad \text { and } \quad \exp F(z)
$$

are the respective egfs for sequences and sets of all lengths $k \geq 0$.

We can, for instance, use these ideas to discover the egf for labeled, rooted trees, which we shall call $T(z)$. Every such tree is an ordered pair $\langle A, B\rangle$ where $A$ is the root node and $B$ is a set of rooted trees (the children of the root). The egf for $A$ is simply $z$, and the egf for $B$ is $\exp T(z)$ according to (2.3); hence we have the well-known relation

$$
T(z)=z e^{T(z)}
$$

Let $U(z)$ be the egf for labeled, unrooted trees. We can represent every rooted tree $T$ on the labels $\{1, \ldots, n\}$ as either an unrooted tree $U$ (if 1 is the root of $T$ ) or as a unordered pair $\{A, B\}$ where $A$ and $B$ are rooted trees (if 1 is not the root of $T$ ). In the latter case, either $A$ or $B$ contains the node 1, say $A$ does; we add an edge from the root of $A$ to the root of $B$. This construction is reversible, hence we have another well-known relation:

$$
T(z)=U(z)+\frac{1}{2} T(z)^{2}
$$

We can now enumerate stopping configurations that contain $k$ unrooted trees in the acyclic components and $l$ rooted trees in the cyclic components: The egf is

$$
T(z)^{l} U(z)^{k} / k !
$$

Summing over $k$ and $l$, the total number of stopping configurations for cycles of length $\geq l$ has the egf

$$
\frac{T(z)^{l}}{1-T(z)} e^{U(z)}
$$


We get all stopping configurations for the uniform model when $l=1$, and for the permutation model when $l=3$.

For our purposes we need additional information provided by a bivariate generating function (bgf), which enumerates stopping configurations by the number of edges as well as the number of vertices. A bgf is a power series

$$
F(w, z)=\sum_{m, n \geq 0} f_{m, n} w^{m} \frac{z^{n}}{n !}
$$

in which $f_{m, n}$ is the number of stopping configurations with $m$ edges and $n$ vertices, weighted by some criterion. Notice that the coefficients are "exponential" in $n$ (i.e., they include a factor $1 / n !)$, but not in $m$; setting $w=1$ converts the bgf into an egf.

The bgf for unrooted trees is $U(w z) / w$, because every tree with $n$ vertices contains $n-1$ edges. Similarly, the bgf for rooted trees is $T(w z) / w$. The bgf for stopping configurations with $k$ unrooted trees and $l$ rooted trees, corresponding to $(2.6)$, is

$$
w^{l}(T(w z) / w)^{l}(U(w z) / w)^{k} / k !=T(w z)^{l}(U(w z) / w)^{k} / k !
$$

because we implicitly associate $l$ additional edges with the edges of the rooted trees. (These are the edges of the cycle.) Summing over $k$ and $l$ gives us the bgf analogous to (2.7) for the set of all stopping configurations with cycle length $\geq l$ :

$$
S_{l}(w, z)=\frac{T(w z)^{l}}{1-T(w z)} e^{U(w z) / w} .
$$

When $l=1$, for example, we obtain the bgf for all configurations in which the uniform model can stop:

$$
\begin{aligned}
S_{1}(w, z)=w z+ & \left(w+2 w^{2}\right) z^{2}+\left(\frac{w+5 w^{2}+9 w^{3}}{2}\right) z^{3} \\
& +\left(\frac{w+9 w^{2}+36 w^{3}+64 w^{4}}{6}\right) z^{4}+\cdots .
\end{aligned}
$$

In particular, when there are $n=3$ vertices the coefficient of $z^{n} / n$ ! is

$$
3 w+15 w^{2}+27 w^{3}
$$

this means that there are 3 stopping configurations in which the uniform model stops after $m=1$ steps, plus 15 in which it stops after $m=2$ steps, plus 27 in which it stops after $m=3$ steps. The 27 with $m=3$ have the following forms:

$\begin{array}{ccccc}6 \text { cases } & 6 \text { cases } & 6 \text { cases } & 6 \text { cases } & 3 \text { cases } \\ & a & b & a & a b \\ & \mid & \mid & \mid & \backslash / \\ \langle a, b, c\rangle & \langle b, c\rangle & \langle a, c\rangle & b & \langle c\rangle\end{array}$


The 15 with $m=2$ include 6 with a 2 -cycle and 9 with a 1-cycle.

Setting $l=3$ in (2.10) gives the bgf for all stopping configurations in the permutation model:

$$
S_{3}(w, z)=w^{3} z^{3}+\left(w^{3}+4 w^{4}\right) z^{4}+\left(\frac{w^{3}+9 w^{4}+25 w^{5}}{2}\right) z^{5}+\cdots
$$

In both models the process must stop after at most $n$ edges have appeared.

3. Probabilities from generating functions. We need to multiply the coefficient of $u^{m} z^{n} / n$ ! in a bgf by a suitable function of $m$ and $n$, in order to compute the probability that a given stopping configuration occurs in the dynamic evolution process.

In the uniform model, a given stopping configuration with $m$ edges $\left\{u_{1}-v_{1}, \ldots\right.$, $\left.u_{m}-v_{m}\right\}$ can arise from exactly $2^{m-1}(m-1)$ ! sequences of ordered pairs $\left\langle x_{1}, y_{1}\right\rangle, \ldots$, $\left\langle x_{m}, y_{m}\right\rangle$. (The values of $x_{m}$ and $y_{m}$ are determined, since they are roots of specific trees in the cycle; the other $m-1$ edges can be permuted in $(m-1)$ ! ways, and there is an additional factor of $2^{m-1}$ because each of these edges can be written as an ordered pair in two ways.) Therefore the probability of obtaining any given $m$-edge, $n$-vertex stopping configuration in this model is $2^{m-1}(m-1) ! / n^{2 m}$.

For example, we can check this calculation when $n=3$, using (2.12):

$$
\frac{3 \cdot 2^{0} \cdot 0 !}{9^{1}}+\frac{15 \cdot 2^{1} \cdot 1 !}{9^{2}}+\frac{27 \cdot 2^{2} \cdot 2 !}{9^{3}}=\frac{1}{3}+\frac{10}{27}+\frac{8}{27} \text {. }
$$

The probabilities sum to 1 , as they should; we note in particular that the process stops in this case after the second step with probability $\frac{10}{27}$.

Given a bgf $F(w, z)=\sum_{m, n} f_{m, n} w^{m} z^{n} / n$ ! as in (2.8), we want to calculate the corresponding probability

$$
\Phi_{n} F=\sum_{m \geq 1} \frac{2^{m-1}(m-1) !}{n^{2 m}} f_{m, n}
$$

for problems of size $n$. The linear functional $\Phi_{n}$ can be obtained in two steps. If we know

$$
F_{n}(w)=n !\left[z^{n}\right] F(w, z)=\sum_{m \geq 1} f_{m, n} w^{m}
$$

then

$$
\Phi_{n} F=\frac{1}{2} \int_{0}^{\infty} e^{-n^{2} t / 2} F_{n}(t) \frac{d t}{t},
$$

because the operation $f(w) \mapsto \frac{1}{2} \int_{0}^{\infty} e^{-n^{2} t / 2} f(t) d t / t$ maps $w^{m}$ into

$$
\frac{1}{2} \int_{0}^{\infty} e^{-n^{2} t / 2} t^{m-1} d t=\frac{2^{m-1}}{n^{2 m}} \int_{0}^{\infty} e^{-u} u^{m-1} d u=\frac{2^{m-1}(m-1) !}{n^{2 m}}
$$


And we do know $F_{n}(w)$, because Cauchy's integral formula gives

$$
F_{n}(w)=\frac{n !}{2 \pi i} \oint F(w, z) \frac{d z}{z^{n+1}}
$$

if we integrate around a small circle enclosing the origin. Therefore $\Phi_{n}$ is determined.

A similar method applies to the permutation model. In this case any stopping configuration with $m$ edges arises from $\frac{1}{2}(m-1)$ ! sequences of pairs $\langle x, y\rangle$ having $x<y$. (The factor $\frac{1}{2}$ comes from the fact that a cycle can be oriented in two ways. Strictly speaking, our definitions impose an ordering on the nodes in the cycle so that exactly half of all stopping configurations with $l \geq 3$ are forbidden.) A given sequence of $m$ edges occurs with probability $1 / N(N-1) \ldots(N-m+1)$, where $N=\left(\begin{array}{l}n \\ 2\end{array}\right)$. Hence the weighting function that converts $m$-edge, $n$-vertex stopping configurations to probabilities in the permutation model is

$$
\frac{(m-1) !}{2 N(N-1) \ldots(N-m+1)}=\frac{1}{2 m\left(\begin{array}{l}
N \\
m
\end{array}\right)} \text {. }
$$

For example, we can check this calculation by looking at the case $n=5$, when there are $N=10$ possible edges. The coefficient of $z^{5} / 5 !$ in $(2.13)$ is $60 w^{3}+540 w^{4}+1500 w^{5}$,
and

$$
\frac{60}{2 \cdot 3 \cdot\left(\begin{array}{c}
10 \\
3
\end{array}\right)}+\frac{540}{2 \cdot 4 \cdot\left(\begin{array}{c}
10 \\
4
\end{array}\right)}+\frac{1500}{2 \cdot 5 \cdot\left(\begin{array}{c}
10 \\
5
\end{array}\right)}=\frac{1}{12}+\frac{9}{28}+\frac{25}{42}=1 \text {. }
$$

The relevant linear functional in the permutation model is

$$
\Psi_{n} F=\sum_{m \geq 1} \frac{f_{m, n}}{2 m\left(\begin{array}{l}
N \\
m
\end{array}\right)}, \quad \text { where } N=\left(\begin{array}{l}
n \\
2
\end{array}\right),
$$

and in this case the integral formula analogous to (3.3) is

$$
\Psi_{n} F=\frac{1}{2} \int_{0}^{\infty} \frac{1}{(1+t)^{N}} F_{n}(t) \frac{d t}{t(1+t)}
$$

(The substitution $u=t /(1+t)$ converts $\int_{0}^{\infty} t^{m-1} d t /(1+t)^{N+1}$ into

$$
\int_{0}^{1} u^{m-1}(1-u)^{N-m} d u=B(N+1-m, m)=\frac{1}{m\left(\begin{array}{l}
N \\
m
\end{array}\right)}
$$

by well-known formulas.) Notice that the kernel factor $(1+t)^{N}=e^{-N\left(t+O\left(t^{2}\right)\right)}$ in (3.5) is analogous to the $e^{-n^{2} t / 2}$ in (3.3).

Our formulas for $\Phi_{n}$ and $\Psi_{n}$ in (3.3) and (3.6) evaluate $F_{n}(w)$ only at positive real values of the parameter $w$. However, $F(w, z)$ is evaluated for (small) complex values of $z$ in (3.4). We can think of $w$ as a positive real parameter in that formula. 
In our applications the bgf $F(w, z)$ actually has the special form

$$
F(w, z)=f(w, T(w z)),
$$

where $T$ is the tree function (2.4). For example, the bgf $S_{l}(w, z)$ of $(2.10)$ is the function $s_{l}(w, T(w z))$ defined by

$$
s_{l}(w, z)=\frac{z^{l}}{1-z} e^{\left(z-z^{2} / 2\right) / w},
$$

because of (2.5). The linear functionals $\Phi_{n}$ and $\Psi_{n}$ can be simplified in such cases because we can "invert" functions of $T$.

Namely, the relation $T(z) e^{-T(z)}=z$ of (2.4) implies that $T$ is the inverse of the function $u e^{-u}$; hence

$$
T\left(u e^{-u}\right)=u
$$

when $|u|$ is small. The contour integral (3.4) now becomes

$$
\begin{aligned}
F_{n}(w) & =\frac{n !}{2 \pi i} \oint f(w, T(w z)) \frac{d z}{z^{n+1}} \\
& =\frac{n ! w^{n}}{2 \pi i} \oint f(w, u) e^{n u}(1-u) \frac{d u}{u^{n+1}} \\
& =n ! w^{n}\left[u^{n}\right] f(w, u) e^{n u}(1-u)
\end{aligned}
$$

if we make the substitution $w z=u e^{-u}$. (This is a special case of a trick often used to prove Lagrange's inversion theorem.)

It turns out that a rescaling of the parameters is quite helpful: We can replace $w$ by $\lambda / n$, thereby introducing a factor $n^{-n}$ that nicely tames the effect of $n !$ in (3.10). An additional factor of $e^{n}$ will reduce the coefficient to polynomial growth; such transformations lead to the following convenient reformulation of the operations described above:

Theorem 1. Let $f(w, T(w z))$ be the bivariate generating function (2.8) for a collection $F$ of stopping configurations. Then the probability that a random graph will lie in $F$, if the graph is constructed by the process described in Section 1 , is $\phi_{\mathrm{n}} f$ for the uniform model and $\psi_{n} f$ for the permutation model, where $\phi_{n}$ and $\psi_{n}$ can be computed as follows:

$$
\begin{aligned}
\phi_{n} f & =\int_{0}^{\infty} f_{n}(\lambda) d \lambda \\
\psi_{n} f & =\int_{0}^{\infty}\left(\frac{e^{\lambda}}{(1+\lambda / n)^{n-1}}\right)^{n / 2} \frac{f_{n}(\lambda)}{1+\lambda / n} d \lambda \\
f_{n}(\lambda) & =\frac{n ! n^{-n} e^{-n \lambda / 2}}{4 \pi i \lambda} \oint f\left(\frac{\lambda}{n}, z\right)(1-z)\left(\frac{\lambda e^{z}}{z}\right)^{n} \frac{d z}{z} .
\end{aligned}
$$


These formulas appear formidable at first glance-there is a contour integral inside a real integral - but we will see that they lead to asymptotic results without great difficulty. The value of $f_{n}(\lambda)$ is nonnegative, and it decreases rapidly to zero when $\lambda$ is greater than 1 because of the factor $e^{-n \lambda / 2}$. The difference between $\phi_{n}$ and $\psi_{n}$ is a rather horrid-looking fudge factor, but we can replace it by its asymptotic value

$$
\left(\frac{e^{\lambda}}{(1+\lambda / n)^{n-1}}\right)^{n / 2} \frac{1}{1+\lambda / n}=e^{\lambda / 2+\lambda^{2} / 4}\left(1-\frac{12 \lambda+3 \lambda^{2}+2 \lambda^{3}}{12 n}+O\left(\frac{\lambda^{2}+\lambda^{6}}{n^{2}}\right)\right)
$$

uniformly for $0 \leq \lambda \leq \frac{1}{2} n$. Thus the two models are roughly the same, except that the permutation model calls for an additional factor of $e^{\lambda / 2+\lambda^{2} / 4}$ in the integral transform. We can take comfort in the fact that some simplification must be possible, since

$$
\begin{array}{ll}
\phi_{n} s_{1}=1 & \text { for all } n \geq 1 ; \\
\psi_{n} s_{3}=1 & \text { for all } n \geq 3 .
\end{array}
$$

(The function $s_{l}$ is defined in (3.8); it yields the probability that the first cycle has length $\geq l$. Thus, formulas (3.15) and (3.16) simply state that the algorithm stops with probability 1 in both models.)

4. Asymptotic distributions. Let's try to get a concrete idea of what the abstract formulas in Theorem 1 mean, by working out some of their simplest consequences. Our goal in this section will be to derive asymptotic formulas for the probability that the cycle length is $\geq l$. Once the methods are understood, more difficult applications will not be much of a challenge.

According to Theorem 1 and equation (3.8), the probability $P_{\geq 1, n}$ that the uniform model produces a cycle of length $\geq l$ is $\int_{0}^{\infty} s_{l, n}(\lambda) d \lambda$, where

$$
\begin{aligned}
s_{l, n}(\lambda) & =\frac{n ! n^{-n} e^{-n \lambda / 2}}{4 \pi i \lambda} \oint z^{l-1} e^{n h(z)} d z, \\
h(z) & =\frac{z-z^{2} / 2}{\lambda}+z-\ln z+\ln \lambda .
\end{aligned}
$$

The contour integral in (4.1) is a polynomial in $\lambda$ of degree $n-1$, and this polynomial is a multiple of $\lambda^{l-1}$, because

$$
\frac{1}{2 \pi i} \oint e^{n\left(\left(z-z^{2} / 2\right) / \lambda+z\right)} \lambda^{n-1} \frac{d z}{z^{n-l+1}}=\left[z^{n-l}\right] \lambda^{n-1} \exp \left(n\left(\frac{z-z^{2} / 2}{\lambda}+z\right)\right) .
$$

For example, when $n=3$ we have

$$
\begin{aligned}
& s_{1,3}(\lambda)=\frac{1}{6}\left(3+5 \lambda+3 \lambda^{2}\right) e^{-3 \lambda / 2} \\
& s_{2,3}(\lambda)=\frac{1}{3}\left(\lambda+\lambda^{2}\right) e^{-3 \lambda / 2} \\
& s_{3,3}(\lambda)=\frac{1}{9} \lambda^{2} e^{-3 \lambda / 2}
\end{aligned}
$$




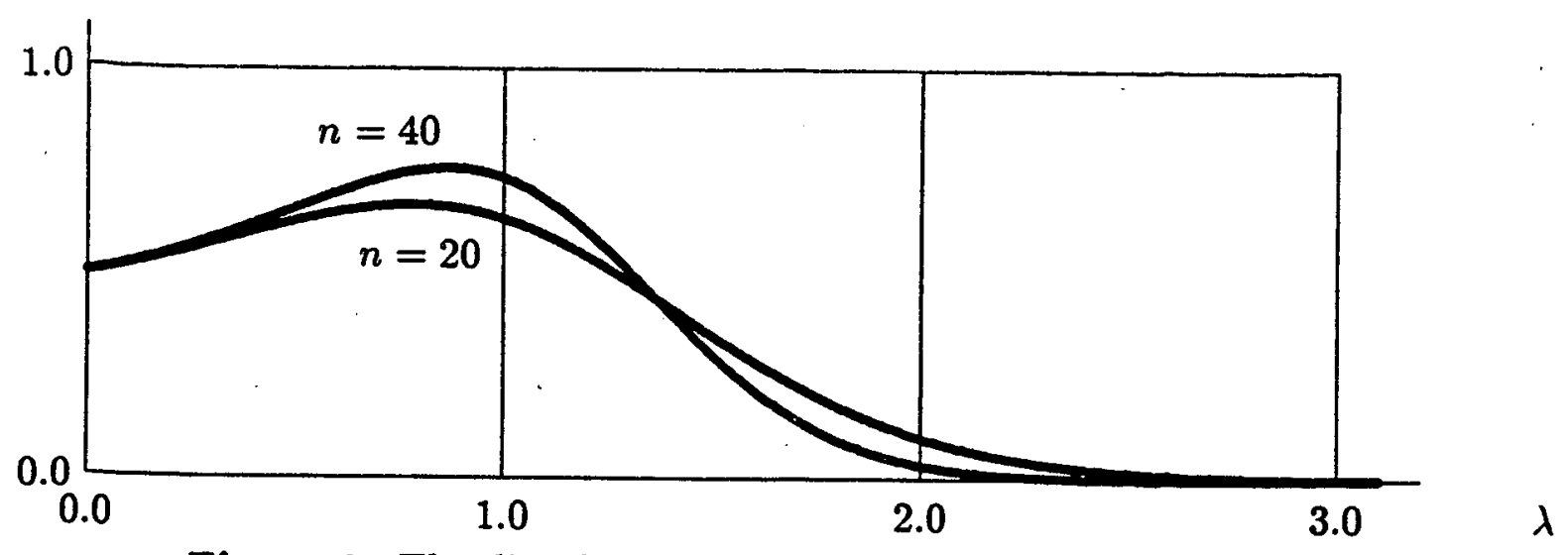

Figure 2. The distribution functions $s_{1, n}(\lambda)$ for $n=20$ and $n=40$.

Integrating over $0 \leq \lambda<\infty$ gives the respective values $1, \frac{28}{81}, \frac{16}{243}$. Hence when $n=3$ the probability that the uniform model produces a cycle of length 1 is $1-\frac{28}{81}=\frac{33}{81}$; the cycle has length 2 with probability $\frac{28}{81}-\frac{16}{243}=\frac{68}{243}$; and it has length 3 with probability $\frac{16}{243}$.

The coefficients of the polynomial part of $s_{l, n}(\lambda)$ always have "mirror symmetry" in the sense that

$$
e^{n \lambda / 2} s_{l, n}(\lambda)=\lambda^{n+l-2} e^{n \lambda^{-1} / 2} s_{l, n}\left(\lambda^{-1}\right)
$$

For example, we have

$$
s_{1,5}(\lambda)=\frac{1}{50}\left(25+70 \lambda+93 \lambda^{2}+70 \lambda^{3}+25 \lambda^{4}\right) e^{-5 \lambda / 2} .
$$

Relation (4.5) is important because it says that we can deduce the value of $s_{l, n}(\lambda)$ for all $\lambda \geq 0$ if we know its value for $0 \leq \lambda \leq 1$. The proof is immediate:

$$
\begin{aligned}
{\left[z^{n-l}\right] \lambda^{n-1} \exp \left(n\left(\frac{z-z^{2} / 2}{\lambda}+z\right)\right) } & =\lambda^{n-1-(n-l)}\left[z^{n-l}\right] \exp \left(n\left(\frac{\lambda z-(\lambda z)^{2} / 2}{\lambda}+\lambda z\right)\right) \\
& =\lambda^{n+l-2}\left[z^{n-l}\right] \lambda^{1-n} \exp \left(n\left(\lambda\left(z-z^{2} / 2\right)+z\right)\right) .
\end{aligned}
$$

Figure 2 shows $s_{1, n}(\lambda)$ when $n=20$ and $n=40$. These functions both yield 1 when integrated from 0 to $\infty$; notice that when $n$ increases, more of the "mass" is concentrated in the range $0 \leq \lambda \leq 1$. In fact, we shall soon prove that $\lim _{n \rightarrow \infty} \int_{0}^{1} s_{1, n}(\lambda) d \lambda=1$. (A "physical" interpretation of this fact appears in Section 8.)

Let us first attempt to find a uniform estimate for $s_{1, n}(\lambda)$ when $0 \leq \lambda<1$. Integrals of the type (4.1) are well suited to the "saddle point method" [Br58, Section 5.7]; hence we investigate the roots of $h^{\prime}(z)=0$ :

$$
\begin{aligned}
h^{\prime}(z) & =\frac{1-z}{\lambda}+1-\frac{1}{z} \\
h^{\prime \prime}(z) & =-\frac{1}{\lambda}+\frac{1}{z^{2}} .
\end{aligned}
$$


There are two saddle points, at $z=\lambda$ and $z=1$. We notice that $h^{\prime \prime}(\lambda)=(1-\lambda) / \lambda^{2}>0$ and $h^{\prime \prime}(1)=(\lambda-1) / \lambda<0$; also $h(1)-h(\lambda)>0$. Hence we want our path of integration to pass vertically through the point $z=\lambda$.

If we integrate around a circle $|z|=r$, where $r$ is any radius between 0 and $(1+\lambda) / 2$, we can show that $\left|e^{h(z)}\right|$ takes its maximum value at $z=r$ and its minimum value at $z=-r$, with no other local maxima or minima. For if $z=r e^{i \theta}$ we have*

$$
\left|e^{h(z)}\right|=e^{\Re h(z)}=e^{f(r, \theta)}
$$

where

$$
f(r, \theta)=\lambda^{-1}\left(r \cos \theta-\frac{1}{2} r^{2} \cos 2 \theta\right)+r \cos \theta-\ln r+\ln \lambda,
$$

and the first derivative

$$
f^{\prime}(r, \theta)=-\lambda^{-1}\left(r \sin \theta-2 r^{2} \sin \theta \cos \theta\right)-r \sin \theta
$$

is zero only when $\sin \theta=0$. Therefore the integrand in (4.1) makes most of its contributions near $\theta=0$. the form

Let us now assume that $\lambda<1$. On the circular path $z=\lambda e^{i \theta}$, equation (4.1) takes

$$
s_{l, n}(\lambda)=\frac{n ! n^{-n} e^{-n \lambda / 2} \lambda^{l-1}}{4 \pi} \int_{-\pi}^{\pi} e^{i l \theta+n h\left(\lambda e^{i \theta}\right)} d \theta .
$$

And by what we have just proved, we can integrate from $-\theta_{0}$ to $\theta_{0}$ instead of from $-\pi$ to $\pi$, for any desired $\theta_{0}<\pi$, using $O\left(\left|\exp \left(n h\left(\lambda e^{i \theta_{0}}\right)\right)\right|\right)$ as an upper bound for the omitted portion of the integral.

The main point of the saddle point method is that $h^{\prime}(\lambda)=0$ and $h^{\prime \prime}(\lambda)>0$, hence $n h\left(\lambda e^{i \theta}\right)$ is approximately $n h(\lambda)-n \lambda^{2} h^{\prime \prime}(\lambda) \theta^{2} / 2$ in the neighborbood of $\theta=0$. Therefore we will be able to estimate the integrand with a formula like $a e^{-n b \theta^{2} / 2}$, plus terms that are asymptotically negligible when $|\theta|$ is small. Let's see what that will buy us, saving the justification for later: If the integrand is replaced by $e^{n h(\lambda)-n h^{\prime \prime}(\lambda) \theta^{2} / 2}$, the integral reduces to

$$
s_{l, n}(\lambda) \approx \frac{n ! n^{-n} e^{n} \lambda^{l-1}}{4 \pi} \int_{-\infty}^{\infty} e^{-n(1-\lambda) \theta^{2} / 2} d \theta
$$

And this is just a multiple of the familiar integral for a normally distributed random variable with mean 0 and variance $1 / n(1-\lambda)$. In general, if $k$ is any nonnegative even integer we have the well-known identity

$$
\int_{-\infty}^{\infty} \theta^{k} e^{-a \theta^{2} / 2} d \theta=2 \int_{0}^{\infty}\left(\frac{2 t}{a}\right)^{k / 2} e^{-t} \frac{d t}{\sqrt{2 a t}}
$$

* We use the notation $\Re z$ for the real part of $z$ and $\Im z$ for the imaginary part. 


$$
\begin{aligned}
& =\frac{2^{(k+1) / 2}}{a^{(k+1) / 2}} \int_{0}^{\infty} t^{(k+1) / 2-1} e^{-t} d t \\
& =\frac{2^{(k+1) / 2} \Gamma((k+1) / 2)}{a^{(k+1) / 2}} \\
& =\frac{\sqrt{2 \pi}}{a^{(k+1) / 2}} \prod_{j=1}^{k / 2}(2 j-1)
\end{aligned}
$$

(The corresponding integral is zero when $k$ is odd.) Therefore our approximation (4.13) simplifies to

$$
s_{l, n}(\lambda) \approx \frac{n ! n^{-n} e^{n} \lambda^{l-1}}{2 \sqrt{2 \pi n(1-\lambda)}} \approx \frac{\lambda^{l-1}}{2 \sqrt{1-\lambda}} .
$$

In fact, it is possible to prove a stronger result, without handwaving:

Lemma 1. If $0 \leq \lambda \leq 1-(\ln n)^{2} / n^{1 / 2}$ and $l \geq 1$, the function $s_{l, n}(\lambda)$ defined in (4.1) satisfies

$$
s_{l, n}(\lambda)=\frac{\lambda^{l-1}}{2 \sqrt{1-\lambda}}+O\left(\frac{l^{2} \lambda^{l-1}}{n(1-\lambda)^{3 / 2}}\right)+O\left(\frac{\lambda^{l-1}}{n(1-\lambda)^{7 / 2}}\right),
$$

uniformly in $\lambda$ and $l$, as $n \rightarrow \infty$.

Proof. On the circle $|z|=\lambda$ the function $h(z)$ is simply

$$
\begin{aligned}
h\left(\lambda e^{i \theta}\right) & =(1+\lambda) e^{i \theta}-\frac{1}{2} \lambda e^{2 i \theta}-i \theta \\
& =1+\frac{1}{2} \lambda+\sum_{k \geq 2}\left(1-\left(2^{k-1}-1\right) \lambda\right) \frac{(i \theta)^{k}}{k !} \\
& =1+\frac{1}{2} \lambda-\frac{1}{2}(1-\lambda) \theta^{2}+i O\left(\theta^{3}\right)+O\left(\theta^{4}\right)
\end{aligned}
$$

where the quantities represented by $O\left(\theta^{3}\right)$ and $O\left(\theta^{4}\right)$ are real. To evaluate (4.12) we want to know the value of

$$
\int_{-\pi}^{\pi} \exp \left(i l \theta+n\left(h\left(\lambda e^{i \theta}\right)-1-\frac{1}{2} \lambda\right)\right) d \theta
$$

and we have observed that it suffices to integrate from $-\theta_{0}$ to $\theta_{0}$, for any convenient value $\theta_{0}$, using the magnitude of the integrand at $\theta_{0}$ to bound the resulting error.

Let $\lambda=1-\mu n^{-1}$, so that $\sqrt{n}(\ln n)^{2} \leq \mu=n(1-\lambda) \leq n$. We will integrate from $-\theta_{0}$ to $\theta_{0}$, where $\theta_{0}=\mu^{-1 / 2} \ln n$. The resulting error will then be exponentially small, because

$$
\begin{aligned}
\left|\exp \left(i l \theta_{0}+n\left(h\left(\lambda e^{i \theta_{0}}\right)-1-\frac{1}{2} \lambda\right)\right)\right| & =\exp \left(-\frac{1}{2} n(1-\lambda) \theta_{0}^{2}+O\left(n \theta_{0}^{4}\right)\right) \\
& =O\left(e^{-(\ln n)^{2} / 2}\right)
\end{aligned}
$$


The substitution $\theta=t \mu^{-1 / 2}$ yields

$$
\begin{aligned}
& \int_{-\theta_{0}}^{\theta_{0}} \exp \left(i l \theta-n(1-\lambda) \theta^{2} / 2+i O\left(n \theta^{3}\right)+O\left(n \theta^{4}\right)\right) d \theta \\
& =\mu^{-1 / 2} \int_{-\ln n}^{\ln n} \exp \left(-t^{2} / 2+O\left(n \mu^{-2} t^{4}\right)\right) \cos \left(l \mu^{-1 / 2} t+O\left(n \mu^{-3 / 2} t^{3}\right)\right) d t \\
& =\mu^{-1 / 2} \int_{-\ln n}^{\ln n} e^{-t^{2} / 2}\left(1+O\left(n \mu^{-2} t^{4}\right)\right) \cos \left(l \mu^{-1 / 2} t+O\left(n \mu^{-3 / 2} t^{3}\right)\right) d t \\
& =\mu^{-1 / 2} \int_{-\infty}^{\infty} e^{-t^{2} / 2}\left(1+O\left(n \mu^{-2} t^{4}\right)\right)\left(1+O\left(l^{2} \mu^{-1} t^{2}\right)+O\left(n^{2} \mu^{-3} t^{6}\right)\right) d t+O\left(e^{-(\ln n)^{2} / 3}\right) \\
& =\sqrt{\frac{2 \pi}{\mu}}\left(1+O\left(n \mu^{-2}\right)+O\left(l^{2} \mu^{-1}\right)+O\left(n^{2} \mu^{-3}\right)\right) .
\end{aligned}
$$

(We are allowed to replace $e^{O(x)}$ by $1+O(x)$ when $x=O(1)$, hence the replacement of $\exp \left(O\left(n \mu^{-2} t^{4}\right)\right)$ by $1+O\left(n \mu^{-2} t^{4}\right)$ is legitimate when $|t| \leq \ln n$. Other estimates made in this derivation, where we replaced $\cos x$ by $1+O\left(x^{2}\right)$ and $(x+y)^{2}$ by $O\left(x^{2}\right)+O\left(y^{2}\right)$, are valid without restrictions on $x$ and $y$.)

The procedure used in the proof of Lemma 1 can be used to obtain as many further terms of the asymptotic expansion as desired (using a computer). For example, the $O$ terms of (4.15) can be shown to equal

$$
\frac{\lambda^{l-1}}{2 \sqrt{1-\lambda}}\left(1+\frac{1}{12 n}-\frac{l^{2}}{2 n(1-\lambda)}-\frac{l(3 \lambda-1)+7 \lambda-1}{2 n(1-\lambda)^{2}}-\frac{5(3 \lambda-1)^{2}}{24 n(1-\lambda)^{3}}\right)
$$

plus terms of lesser order. However, we reach a point of diminishing returns in these estimates when $\lambda$ becomes larger than $1-n^{-1 / 3}$ or when $l$ becomes larger than $n^{1 / 2}$.

Lemma 2. If $\lambda \geq 1+(\ln n)^{2} / n^{1 / 2}$ and $l \geq 1$, the function $s_{l, n}(\lambda)$ defined in (4.1) satisfies

$$
s_{l, n}(\lambda)=\frac{e^{-n(\lambda-1 / \lambda) / 2} \lambda^{n}}{2 \sqrt{\lambda(\lambda-1)}}\left(1+O\left(\frac{l^{2} \lambda}{n(\lambda-1)}\right)+O\left(\frac{\lambda^{3}}{n(\lambda-1)^{3}}\right)\right)
$$

uniformly in $\lambda$ and $l$, as $n \rightarrow \infty$.

Proof. We could prove this by contour integration, using an argument almost identical to that in Lemma 1 but choosing the other saddle point and integrating around $|z|=1$. But (4.18) is actually an immediate consequence of Lemma 1 and the reflection law (4.5).

For fixed $\lambda>1$, equation (4.18) implies that $s_{l, n}(\lambda)$ decreases exponentially to zero as $n \rightarrow \infty$, because $\lambda-1 / \lambda>2 \ln \lambda$. (If $\lambda=e^{t}$, we always have $e^{t}-e^{-t}>2 t$.) On the 
other hand, the difference between $\lambda-1 / \lambda$ and $2 \ln \lambda$ is of order $(\lambda-1)^{3}$, so formula (4.18) says that

$$
s_{l, n}\left(1+\epsilon n^{-1 / 3}\right)=\frac{e^{-\epsilon^{3} / 6} n^{1 / 6}}{2 \sqrt{\epsilon}}\left(1+O\left(\frac{l^{2}}{n^{2 / 3} \epsilon}\right)+O\left(\frac{1}{\epsilon^{3}}\right)+O\left(\frac{\epsilon+\epsilon^{4}}{n^{1 / 3}}\right)\right),
$$

when $n^{-1 / 6}(\ln n)^{2} \leq \epsilon<\frac{1}{2} n^{1 / 3}$. Thus $s_{l, n}\left(1+n^{-1 / 3}\right)$ is of order $n^{1 / 6}$, but the nearby value $s_{l, n}\left(1+n^{-1 / 3} \ln n\right)$ is already exponentially small. In other words $s_{l, n}(\lambda)$ is unbounded as $n \rightarrow \infty$, but it decreases very rapidly when $\lambda$ passes 1 .

Lemma 1 tells us that $s_{l, n}\left(1-n^{1 / 3}\right)$ is of order $n^{1 / 6}$ and that $s_{l, n}\left(1-n^{-1 / 3} \ln n\right) \sim$ $\frac{1}{2} n^{1 / 6}(\ln n)^{-1 / 2}$. But the error estimates in both Lemmas 1 and 2 blow up when $\lambda$ is near 1 , because the two saddle points at $\lambda$ and 1 come together; indeed, we have $h^{\prime}(1) \approx 0$ and $h^{\prime \prime}(1) \approx 0$ but $h^{\prime \prime \prime}(1) \neq 0$ when $\lambda \approx 1$, so the magnitude of $e^{h(z)}$ near $z=1$ has a graph that looks like a three-legged saddle-as used perhaps by Martian horsemen. A third lemma closes the gap in our knowledge by focussing on the region near $\lambda=1$ :

Lemma 3. If $|\mu| \leq n^{1 / 12}$ and $l \leq n^{1 / 4}$, the function $s_{l, n}(\lambda)$ defined in (4.1) satisfies

$$
s_{l, n}\left(e^{-\mu n^{-1 / 3}}\right)=\frac{n^{1 / 6}}{\sqrt{8 \pi} i} \int_{-i \infty}^{i \infty} e^{P(\mu, s)} d s\left(1+O\left(\frac{\beta(\mu, n) l+\beta(\mu, n)^{4}}{n^{1 / 3}}\right)\right)
$$

uniformly in $\mu$ and $l$ as $n \rightarrow \infty$, where

$$
\begin{aligned}
& P(\mu, s)=\frac{(\mu+2 s)(\mu-s)^{2}}{6}, \\
& \beta(\mu, n)=|\mu|+\ln n .
\end{aligned}
$$

Proof. Let $\nu=n^{-1 / 3}$. We will integrate on the circle $|z|=e^{-\alpha \nu}$, where $\alpha$ is the positive solution to

$$
\mu=\alpha-\alpha^{-1}
$$

this radius comes close enough to the saddle point for our purposes. The integrand on $|x|=e^{-\alpha \nu}$ has the nice monotonicity property described in connection with (4.11), because $e^{-\alpha \nu}<\left(1+e^{-\mu \nu}\right) / 2=(1+\lambda) / 2$.

A straightforward calculation now proves that, for any $s$,

$$
-\frac{1}{2} e^{-\mu \nu}+h\left(e^{-s \nu}\right)=1+\nu^{3} P(\mu, s)+R^{\prime},
$$

where the remainder term is

$$
\begin{aligned}
R^{\prime} & =\sum_{k \geq 4}\left((\mu-s)^{k}-\frac{1}{2}(\mu-2 s)^{k}+(-s)^{k}+\frac{1}{2} \mu^{k}\right) \frac{\nu^{k}}{k !} \\
& =\sum_{k \geq 4} \frac{O((|\mu|+|s|) \nu)^{k}}{k !}=O((|\mu|+|s|) \nu)^{4}
\end{aligned}
$$


uniformly in any region where $(|\mu|+|s|) \nu$ is bounded. The terms in $\nu$ and $\nu^{2}$ have cancelled out beautifully from the right-hand side of (4.23), thereby making the asymptotic behavior simple when we multiply by $n=\nu^{-3}$.

Formula (4.1), with $z=e^{-\alpha \nu+i \theta}$ and $\theta=t \nu$, becomes

$$
s_{l, n}\left(e^{-\mu \nu}\right)=\frac{n ! n^{-n} e^{n} \nu}{4 \pi e^{-\mu \nu}} \int_{-\pi n^{1 / 3}}^{\pi n^{1 / 3}} \exp \left((i t-\alpha) l \nu+P(\mu, \alpha+i t)+O\left((|\mu|+1+|t|)^{4} \nu\right)\right) d t
$$

because $\alpha \leq|\mu|+1$. We can restrict the range of integration to $|t| \leq \ln n$ as in Lemma 1 , incurring only an exponentially small relative error, because the real part of the exponent $P(\mu, \alpha-i t)-P(\mu, \alpha)$ is $-\frac{1}{2}\left(\alpha+\alpha^{-1}\right)(\ln n)^{2} \leq-\frac{1}{2}(\ln n)^{2}$ when $|t|=\ln n$, while the $O$ term is $O\left((|\mu|+1+\ln n)^{4} \nu\right)=O(1)$ by our assumption that $|\mu|=O\left(n^{1 / 12}\right)$. The integrand can now be replaced by $e^{P(\mu, \alpha-i t)}$ times $1+O((|\mu|+\ln n) l \nu)+O\left((|\mu|+\ln n)^{4} \nu\right)$, after which we can extend the integration from $-\infty$ to $\infty$. Finally, the substitution $s=\alpha-i t$ gives the stated result (4.19), except that the integral runs from $\alpha-i \infty$ to $\alpha+i \infty$ instead of simply from $-i \infty$ to $i \infty$. The path of integration can be shifted to the left, because the integrand is exponentially small when $s= \pm i R+a$ and $R \rightarrow \infty$, if $a$ is any positive constant.

The integral (4.19) is investigated further in the appendix below, where the following result is derived as a special case of a general series expansion:

$$
s_{l, n}(1) \sim \frac{\Gamma\left(\frac{1}{3}\right)}{3^{1 / 6} \sqrt{8 \pi}} n^{1 / 6}
$$

5. Distribution of cycle lengths. We can now combine the three lemmas with Theorem 1 and obtain the limiting probability distribution of cycle lengths:

Theorem 2. For fixed $l$ as $n \rightarrow \infty$, the graph evolution procedure of Section 1 generates a first cycle of length $l$ with probability

$$
P_{l, n}=\frac{1}{3} \prod_{k=1}^{l-1}\left(\frac{2 k}{2 k+3}\right)+O\left(n^{-1 / 6}\right), \quad l \geq 1,
$$

in the uniform model, and with probability

$$
\widehat{P}_{l, n}=\frac{1}{2} \int_{0}^{1} \lambda^{l-1} \sqrt{1-\lambda} e^{\lambda / 2+\lambda^{2} / 4} d \lambda+O\left(n^{-1 / 6}\right), \quad l \geq 3
$$

in the permutation model.

Proof. In the uniform model we have

$$
P_{l, n}=\int_{0}^{\infty}\left(s_{l, n}(\lambda)-s_{l+1, n}(\lambda)\right) d \lambda
$$


so it suffices to determine $\int_{0}^{\infty} s_{l, n}(\lambda) d \lambda$. The integral for $\lambda \geq 1-n^{-1 / 3}$ is $O\left(n^{-1 / 6}\right)$, by Lemmas 4.2 and 4.3. Therefore we may restrict consideration to the interval $0 \leq \lambda \leq$ $1-n^{-1 / 3}$, when we find that the total error in (4.15) is

$$
n^{-1} \int_{0}^{1-n^{-1 / 3}} O\left((1-\lambda)^{-7 / 2}\right) d \lambda=n^{-1} O\left(n^{5 / 6}\right)=O\left(n^{-1 / 6}\right)
$$

The integral from $1-n^{-1 / 3}$ to 1 of $(1-\lambda)^{-1 / 2}$ is also $O\left(n^{-1 / 6}\right)$; hence

$$
P_{\geq l, n}=\int_{0}^{\infty} s_{l, n}(\lambda) d \lambda=\frac{1}{2} \int_{0}^{1} \frac{\lambda^{l-1} d \lambda}{\sqrt{1-\lambda}}+O\left(n^{-1 / 6}\right)
$$

And this is a Beta integral,

$$
P_{\geq l, n}=\frac{1}{2} B\left(l, \frac{1}{2}\right)=\frac{1}{2} \frac{\Gamma(l) \Gamma\left(\frac{1}{2}\right)}{\Gamma\left(l+\frac{1}{2}\right)}=\prod_{k=1}^{l-1}\left(\frac{2 k}{2 k+1}\right)
$$

The difference $P_{\geq l, n}-P_{\geq l+1, n}$ is, similarly, $\frac{1}{2} B\left(l, \frac{3}{2}\right)$, and we obtain (5.1). Equation (5.2) follows from (3.12) and (3.14).

Thus the cycle lengths approach a stationary distribution, without any normalization. Formula (5.2) was first obtained (without the error bound) by Svante Janson [Ja87], using a general theory of Poisson processes, and independently by Béla Bollobás [Bo89], using the theory of martingales.

Since the extra factor $e^{\lambda / 2+\lambda^{2} / 4}$ lies between 1 and $e^{3 / 4} \approx 2.11700002$ for $0 \leq \lambda \leq 1$, both probabilities $P_{l, n}$ and $\hat{P}_{l, n}$ have the same order of growth as $l$ increases. Indeed, let

$$
p_{l}=\frac{1}{3} \prod_{k=1}^{l-1}\left(\frac{2 k}{2 k+3}\right)=\frac{1}{2} \int_{0}^{1} \lambda^{l-1} \sqrt{1-\lambda} d \lambda ; \quad \hat{p}_{l}=\frac{1}{2} \int_{0}^{1} \lambda^{l-1} \sqrt{1-\lambda} e^{\lambda / 2+\lambda^{2} / 4} d \lambda .
$$

Then we can write $\lambda / 2+\lambda^{2} / 4=3 / 4+O(\lambda-1)$, obtaining

$$
p_{l}=\frac{\sqrt{\pi}}{4 l^{3 / 2}}+O\left(l^{-5 / 2}\right) ; \quad \hat{p}_{l}=\frac{\sqrt{\pi} e^{3 / 4}}{4 l^{3 / 2}}+O\left(l^{-5 / 2}\right)
$$

In both cases the average value $\Sigma_{l} p_{l}$ is infinite; therefore the expected cycle length must be unbounded as $n \rightarrow \infty$.

The limiting probabilities $p_{l}$ for the uniform model obey simple recurrence relations:

$$
p_{l+1}=\frac{2 l}{2 l+3} p_{l} ; \quad p_{\geq l+1}=\frac{2 l}{2 l+1} p_{\geq l}=2 l p_{l}
$$


Hence it is natural to wonder if the corresponding numbers $\hat{p}_{l}$ and $\hat{p}_{\geq 1}$ for the permutation model satisfy similar recurrences, and in fact they do. First we note that

$$
\hat{p}_{\geq 3}=\frac{1}{2} \int_{0}^{1} \frac{\lambda^{2}}{\sqrt{1-\lambda}} e^{\lambda / 2+\lambda^{2} / 4} d \lambda=-\left.\sqrt{1-\lambda} e^{\lambda / 2+\lambda^{2} / 4}\right|_{0} ^{1}=1 .
$$

A similar integration shows that

$$
\hat{p}_{\geq l+1}=(2 l-4) \hat{p}_{l-2},
$$

and it follows that we have the recurrence

$$
\hat{p}_{l+2}=2(l-1) \hat{p}_{l-1}-2 l \hat{p}_{l} .
$$

Is there a "simple" graph-theoretic explanation of (5.10)?

Setting $l=5$ in $(5.10)$ yields

$$
\hat{p}_{5}=1-7 \hat{p}_{3}-\hat{p}_{4},
$$

hence the values of $\hat{p}_{l}$ can all be expressed in the form $a_{l}+b_{l} \hat{p}_{3}+c_{l} \hat{p}_{4}$ where $a_{l}, b_{l}$, and $c_{l}$ are integers. Recurrence (5.11) is numerically unstable, but we can obtain accurate values

$$
\begin{aligned}
& \hat{p}_{3}=0.12160822171448358918, \\
& \hat{p}_{4}=0.08491509952633599860,
\end{aligned}
$$

by calculating $\hat{p}_{l}$ and $\hat{p}_{l+1}$ accurately for some large $l$ and then solving backwards.

Do the fundamental quantities $s_{l, n}(\lambda)$ defined in (4.1) obey a recurrence relation? Yes, but it is a bit more complicated: We have

$$
s_{l+2, n}(\lambda)=(1+\lambda) s_{l+1, n}(\lambda)-\lambda\left(1-\frac{l}{n}\right) s_{l, n}(\lambda) .
$$

This relation follows since $s_{l+1, n}(\lambda)$ has the form

$$
\frac{f(n, \lambda)}{2 \pi i} \oint z^{i} e^{n h(z)} d z
$$

where $f(n, \lambda)$ does not depend on $l$. Differentiating the integrand with respect to $z$ yields a function with nothing but zero residues, hence

$$
\begin{aligned}
0 & =\frac{f(n, \lambda)}{2 \pi i} \int z^{l} e^{n h(z)}\left(\frac{l}{z}+n h^{\prime}(z)\right) d z \\
& =l s_{l, n}(\lambda)+n\left(\frac{s_{l+1, n}(\lambda)-s_{l+2, n}(\lambda)}{\lambda}+s_{l+1, n}(\lambda)-s_{l-1, n}(\lambda)\right) .
\end{aligned}
$$


The recurrence (5.15) can be used to calculate $s_{l, n}(\lambda)$ "backwards," starting with the values

$$
s_{n+1, n}(\lambda)=0, \quad s_{n, n}(\lambda)=\frac{1}{2} n ! n^{-n} \lambda^{n-1} e^{-n \lambda / 2},
$$

and working down to $s_{1, n}(\lambda)$. This does not appear to lead to any simple consequences about the asymptotic behavior of $s_{l, n}(\lambda)$. We can, however, use (5.15) to prove by induction that the coefficients of the polynomials $\left(s_{l, n}(\lambda)-s_{l+1}(\lambda)\right) e^{n \lambda / 2}$ are nonnegative. Furthermore, (5.15) implies the remarkable identity

$$
\sum_{l \geq 1} l s_{l, n}(\lambda)=n\left(s_{1, n}(\lambda)-\lambda^{-1} s_{2, n}(\lambda)\right)
$$

which can be used to study the variance of the cycle lengths.

6. The average cycle length. We have seen in Section 3 how to set up a bivariate generating function $F(w, z)$ for a set of stopping configurations, thereby allowing us to compute the probability $\Phi_{n} F$ that such configurations occur in a graph of $n$ vertices. But we can, of course, also use $\Phi_{n} F$ to compute expected values, if $F(w, z)$ is a bgf in which each stopping configuration has been multiplied by a weight representing the random variable in question.

For example, $T(w z)^{l} e^{U(w z) / w}$ is the bgf for stopping configurations with cycles of length $l$, hence

$$
\begin{aligned}
A(w, z) & =\left(T(w z)+2 T(w z)^{2}+3 T(w z)^{3}+\cdots\right) e^{U(w z) / w} \\
& =\frac{T(w z)}{(1-T(w z))^{2}} e^{U(w z) / w}
\end{aligned}
$$

is a bgf such that $\Phi_{n} A$ is the expected cycle length in the uniform model. According to Theorem 1 , this expected cycle length is

$$
\int_{0}^{\infty} a_{n}(\lambda) d \lambda, \quad a_{n}(\lambda)=\frac{n ! n^{-n} e^{-n \lambda / 2}}{4 \pi i \lambda} \oint \frac{1}{1-z} e^{n h(z)} d z
$$

where $h(z)$ is the familiar function of (4.2). Notice that we have

$$
a_{n}(\lambda)=\sum_{l \geq 1} s_{l, n}(\lambda)=\sum_{l=1}^{n} s_{l, n}(\lambda)
$$

Since $s_{l, n}(\lambda)$ is exponentially small for $\lambda \geq 1+n^{-1 / 3} \ln n$, we need not consider large values of $\lambda$. However, the presence of $1-z$ in the denominator of (6.2) means that values of $a_{n}(\lambda)$ near $\lambda=1$ will be crucial. 


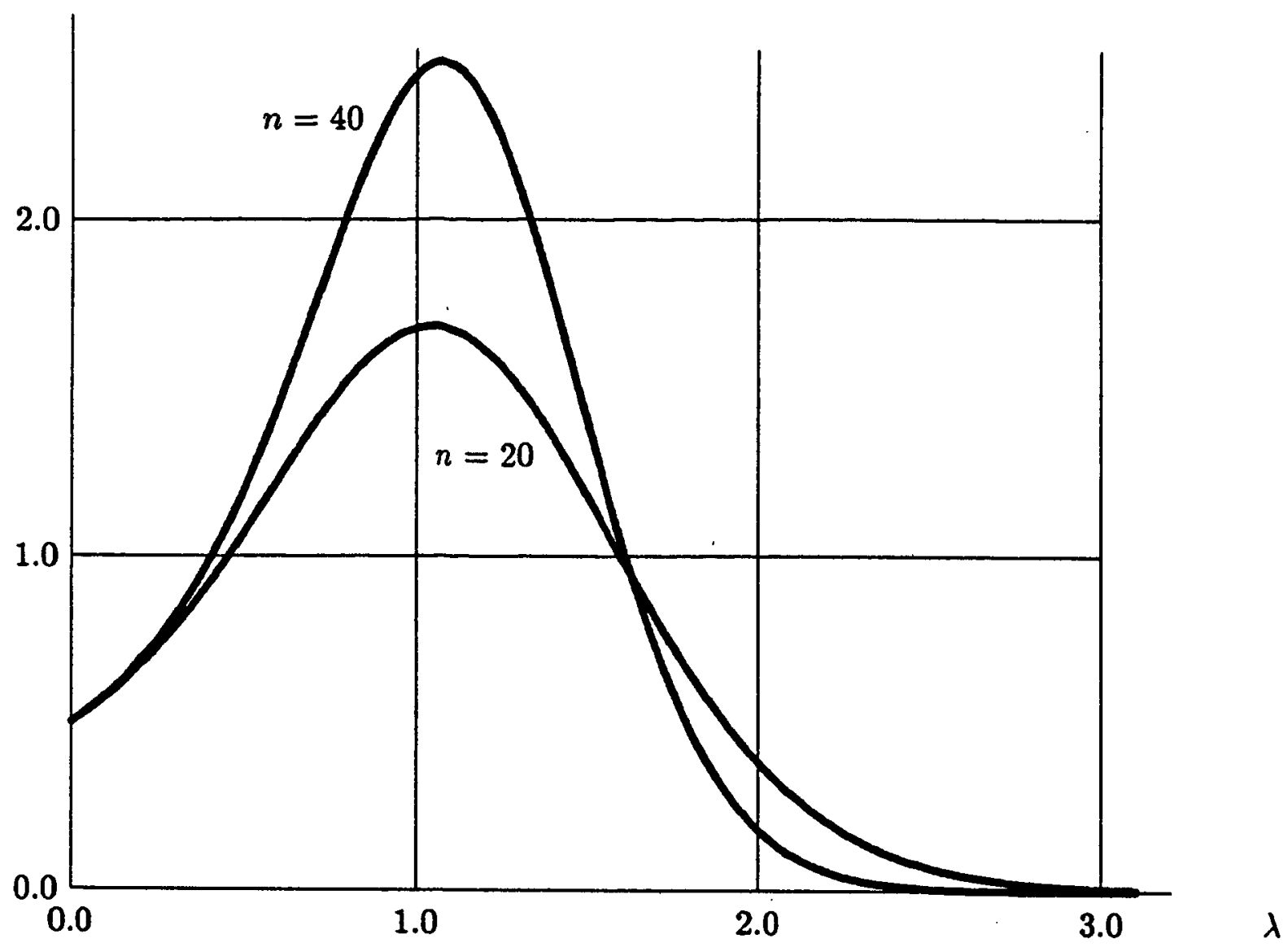

Figure 3. The weighted distribution functions $a_{n}(\lambda)$ for $n=20$ and $n=40$.

A slight modification to the proof of Lemma 1 shows that the asymptotic formula

$$
a_{n}(\lambda)=\frac{1}{2(1-\lambda)^{3 / 2}}+O\left(\frac{1}{n(1-\lambda)^{9 / 2}}\right)
$$

holds uniformly for $0 \leq \lambda \leq 1-n^{-1 / 2}(\ln n)^{2}$ as $n \rightarrow \infty$. If we integrate this quantity as $\lambda$ varies from 0 to $1-n^{-1 / 3} \ln n$, say, we get

$$
\frac{n^{1 / 6}}{\sqrt{\ln n}}+O\left(\frac{n^{1 / 6}}{(\ln n)^{7 / 2}}\right)
$$

hence we may conclude that the value of $a_{n}(\lambda)$ is negligible except when $|\lambda-1| \leq n^{-1 / 3} \ln n$, if we can show that the integral of $a_{n}(\lambda)$ over that range is of order $n^{1 / 6}$.

Figure 3 shows the behavior of $a_{n}(\lambda)$ for $n=20$ and $n=40$. As $n$ increases, the function has sharper and sharper peaks, apparently reaching a maximum when $\lambda$ is very slightly greater than 1 .

The contour integral that arises when $\lambda$ is near 1 is just like the integral we studied in Lemma 3, except that there is an additional factor $(1-z)^{-1}$. If we set $z=e^{(i t-\alpha) \nu}$ as 
in that lemma, we have

$$
\frac{1}{1-z}=-\sum_{k \geq 0}(i t-\alpha)^{k-1} \nu^{k-1} \frac{B_{k}}{k !}=\frac{n^{1 / 3}}{\alpha-i t}+O(1),
$$

uniformly in $\alpha$ and $t$, provided that $|\alpha-i t| \leq 6 n^{1 / 3}$, since the series converges for $|\alpha-i t| \leq$ $2 \pi n^{1 / 3}$. Therefore the calculations of Lemma 3 can be applied almost without change, and we obtain

$$
a_{n}\left(e^{-\mu n^{-1 / 3}}\right)=\frac{n^{1 / 2}}{\sqrt{8 \pi} i} \int_{1-i \infty}^{1+i \infty} e^{P(\mu, s)} \frac{d s}{s}+O\left(n^{1 / 8}\right),
$$

uniformly over all $\mu$ such that $|\mu| \leq n^{1 / 12}$. (The integrand now has a pole at $s=0$, so we are no longer allowed to shift the path of integration to the imaginary axis. But we can integrate for $c-i \infty$ to $c+i \infty$ for any $c>0$.)

Finally we can compute the asymptotic path length, proving the formula claimed in the introduction:

Theorem 3. The expected length of the first cycle in an evolving graph is $K n^{1 / 6}+O\left(n^{1 / 8}\right)$ in the uniform model, and $e^{3 / 4} K n^{1 / 6}+O\left(n^{1 / 8}\right)$ in the permutation model, where

$$
K=\frac{1}{\sqrt{8 \pi} i} \int_{-\infty}^{\infty} \int_{1-i \infty}^{1+i \infty} e^{(\mu+2 s)(\mu-s)^{2} / 6} \frac{d s}{s} d \mu .
$$

Proof. Setting $\lambda=e^{-\mu n^{-1 / 3}}, \lambda_{1}=e^{-n^{-1 / 4}}$, and $\lambda_{2}=e^{n^{-1 / 4}}$, we have

$$
\int_{\lambda_{1}}^{\lambda_{2}} a_{n}(\lambda) d \lambda=\frac{n^{1 / 8}}{\sqrt{8 \pi} i} \int_{\mu_{2}}^{\mu_{1}} e^{-\mu n^{-1 / 8}} \int_{1-i \infty}^{1+i \infty} e^{P(\mu, 0)} \frac{d s}{s} d \mu+O\left(n^{-1 / 12}\right),
$$

where $\mu_{1}=n^{1 / 12}$ and $\mu_{2}=-n^{1 / 12}$. When $\mu$ is between $\mu_{2}$. and $\mu_{1}$, the integrand factor $\exp \left(-\mu n^{-1 / 3}\right)$ is $1+O\left(n^{-1 / 4}\right)$, so we can ignore it. Thus we obtain an integral whose integrand matches (6.8).

This integrand is exponentially small as $\mu \rightarrow-\infty$, and we will prove in the appendix that it is $O\left(\mu^{-3 / 2}\right)$ as $\mu \rightarrow \infty$. Extending the integral from $-\infty$ to $\infty$, instead of from $\mu_{2}$ to $\mu_{1}$, therefore introduces an error of $n^{1 / 6} O\left(\mu_{1}^{-1 / 2}\right)=O\left(n^{1 / 8}\right)$. To obtain the total expected length $\int_{0}^{\infty} a_{n}(\lambda) d \lambda$, we must add $\left(\int_{0}^{\lambda_{1}}+\int_{\lambda_{2}}^{\infty}\right) a_{n}(\lambda) d \lambda$; this gives a further error of $O\left(n^{1 / 8}\right)$, by (6.4), so we have established the result claimed for the uniform model.

The permutation model requires an additional factor

$$
\exp \left(\frac{1}{2} \lambda+\frac{1}{4} \lambda^{2}\right)=\exp \left(\frac{3}{4}+O\left(|\mu| n^{-1 / 3}\right)\right)
$$

which is treated similarly. There also is a (negligible) factor $e^{2(\alpha-i t) \nu}$ in the inner integral, because the numerator of the bgf in (6.1) must be changed from $T(w z)$ to $T(w z)^{3}$ in order to get the expected value of $l-2$. 
7. Additional statistics. To find the variance of the cycle length, we can compute

$$
\int_{0}^{\infty} b_{n}(\lambda) d \lambda, \quad B(w, z)=\frac{T(w z)}{(1-T(w z))^{3}} e^{U(w z) / w},
$$

which is the expected value of $\frac{1}{2} l(l-1)$. We get $b_{n}(\lambda)$ from $a_{n}(\lambda)$ by essentially changing (6.6) to

$$
\frac{n^{2 / 3}}{(\alpha-i t)^{2}}+O\left(|\alpha-i t| n^{1 / 3}\right)
$$

The result, since $s=\alpha-i t$, is to multiply the formula for $\int_{0}^{\infty} a_{n}(\lambda) d \lambda$ by $n^{1 / 3}$, and to change the constant of proportionality by replacing $s$ by $s^{2}$ in the denominator of (6.8). Thus the expected value of $\frac{1}{2} l(l-1)$ is of order $n^{1 / 2}$; the standard deviation is therefore asymptotically proportional to $n^{1 / 4}$, somewhat greater than the mean.

In general, if we have a bgf of the form

$$
C_{l, k}(w, z)=\frac{T(w z)^{l}}{(1-T(w z))^{k}} e^{U(w z) / w}, \quad k>\frac{3}{2},
$$

where $l$ is fixed as $n \rightarrow \infty$, the resulting value of $\int_{0}^{\infty} c_{l, k, n}(\lambda) d \lambda$ will be of order $n^{(2 k-3) / 6}$, by the same argument.

Therefore we can grind out more facts by setting up appropriate bgfs. Let us introduce (temporarily) a trivariate generating function

$$
D_{l}(\zeta, w, z)=\frac{T(\zeta w z)^{l}}{1-T(\zeta w z)} e^{U(w z) / w},
$$

in which the coefficient of $\zeta^{j} w^{m} z^{n} / n$ ! is the number of stopping configurations with cycles of length $\geq l$ having $m$ edges and $n$ vertices, with $j$ vertices in the cyclic component. If we take the partial derivative with respect to $\zeta$ and then set $\zeta=1$, we get a bgf for the expected value of $j$, namely

$$
D_{l}^{\prime}(1, w, z)=\frac{T(w z)^{l+1}}{(1-T(w z))^{2}}\left(\frac{l}{T(w z)}+\frac{1}{1-T(w z)}\right) e^{U(w z) / w} .
$$

(This follows from the well-known relation

$$
T^{\prime}(z)=\frac{T(z)}{z(1-T(z))}
$$

a consequence of (2.4).) Another derivative gives the expected value of $j(j-1)$ and introduces another $(1-T(w z))^{2}$ in the denominator. Therefore (7.3) applies and we can state: 
Corollary 1. The expected size of the first cyclic component in an evolving graph on $n$ vertices is asymptotically proportional to $n^{1 / 2}$, and the standard deviation is of or$\operatorname{der} n^{7 / 12}$.

Proof. Take $l=1$ in (7.4) for the uniform model and $l=3$ for the permutation model; use $k=3$ in (7.3) for the mean and $k=5$ for the variance.

A similar derivation, with $T(\zeta w z) T(w z)^{l} e^{U(w z) / w} /(1-T(w z))$ in place of (7.4), shows that the expected number of vertices in the tree that leads into the first vertex $x_{m}$ of the first cycle is the same as the expected length of that cycle. The same holds for any individual tree in the cyclic component. Thus the cyclic component consists of $\Theta\left(n^{1 / 6}\right)$ trees, on the average, each of which has $\Theta\left(n^{1 / 6}\right)$ vertices, on the average; a dependency between these two statistics causes the overall expected size to be $\Theta\left(n^{1 / 2}\right)$.

We can find the limiting distribution of the number of vertices in the first cyclic component by considering the coefficient of $\zeta^{j}$ in (7.4). Indeed, we have

$$
D_{1}(\zeta, w, z)=\sum_{j \geq 1} \zeta^{j} \frac{j^{j}}{j !}(w z)^{j} e^{U(w z) / w}
$$

and we can write this as a function of $w$ and $T(w z)$ by using identity (2.4), which says that $w z=T(w z) e^{-T(w z)}$. Our general method now tells us to evaluate the integral

$$
\oint\left(z e^{-z}\right)^{j} \frac{1-z}{z} e^{n h(z)} d z
$$

asymptotically as $n \rightarrow \infty$. We find as before that the only relevant contributions occur when $\lambda<1$, and an argument like that of Theorem 2 shows that a proper probability distribution appears in the limit:

Corollary 2. For fixed $j$ as $n \rightarrow \infty$, the random graph evolution procedure generates a first cyclic component of size $j$ with probability

$$
\theta_{j, n}=\frac{1}{2} \frac{j^{j}}{j !} \int_{0}^{1} \lambda^{j-1} e^{-j \lambda} \sqrt{1-\lambda} d \lambda+O\left(n^{-1 / 6}\right), \quad j \geq 1,
$$

in the uniform model, and with probability

$$
\begin{gathered}
\hat{\theta}_{j, n}=\frac{1}{2} \frac{j^{j-2}(j-1)(j-2)}{j !} \int_{0}^{1} \lambda^{j-1} e^{-j \lambda+\lambda / 2+\lambda^{2} / 4} \sqrt{1-\lambda} d \lambda \\
+O\left(n^{-1 / 6}\right), \quad j \geq 3,
\end{gathered}
$$

in the permutation model. 
These limiting probabilities $q_{j}$ and $\hat{q}_{j}$ sum to 1 . We have, for example,

$$
\begin{aligned}
\sum_{j \geq 1} q_{j} & =\frac{1}{2} \sum_{j \geq 1} \frac{j^{j}}{j !} \int_{0}^{1} \lambda^{j-1} e^{-j \lambda} \sqrt{1-\lambda} d \lambda \\
& =\frac{1}{2} \int_{0}^{1} \lambda^{-1}\left(\sum_{j \geq 1} \frac{\left(j \lambda e^{-\lambda}\right)^{j}}{j !}\right) \sqrt{1-\lambda} d \lambda \\
& =\frac{1}{2} \int_{0}^{1} \lambda^{-1} \frac{T\left(\lambda e^{-\lambda}\right)}{1-T\left(\lambda e^{-\lambda}\right)} \sqrt{1-\lambda} d \lambda=\frac{1}{2} \int_{0}^{1}(1-\lambda)^{-1 / 2} d \lambda=1 .
\end{aligned}
$$

Both $q_{j}$ and $\hat{q}_{j}$ are of order $j^{-5 / 4}$ as $j$ grows; indeed, the substitution $\lambda=1-$ $\sqrt{2 t / n}$ shows that $q_{j}=c j^{-5 / 4}+O\left(j^{-7 / 4}\right)$ and $\hat{q}_{j}=e^{3 / 4} c j^{-5 / 4}+O\left(j^{-7 / 4}\right)$, where $c=$ $2^{-7 / 4} \pi^{-1 / 2} \Gamma\left(\frac{3}{4}\right)$. We have seen in Corollary 1 that the expected value of the component size is unbounded. Here is a table of approximate probabilities when $j$ is small:

$$
\begin{aligned}
q_{1} & =.23096 & \hat{q}_{3} & =.01804 \\
q_{2} & =.09501 & \hat{q}_{4} & =.02181 \\
q_{3} & =.05649 & \hat{q}_{5} & =.02153 \\
q_{4} & =.03909 & \hat{q}_{6} & =.02015 \\
q_{10} & =.01214 & \hat{q}_{10} & =.01436 \\
q_{20} & =.00504 & \hat{q}_{20} & =.00754
\end{aligned}
$$

The value of $q_{1}$ is $\frac{1}{2}+\frac{1}{4} e^{-1} \sqrt{\pi} i \operatorname{erf}(i)$, according to MACSYMA.

To get the expected value of $m$, the number of edges, we can use the fact that $n-m$ is the number of acyclic components. The relevant trivariate generating function is

$$
E_{l}(\zeta, w, z)=\frac{T(w z)^{l}}{1-T(w z)} e^{\zeta U(w z) / w}
$$

and we have

$$
\begin{aligned}
E_{l}^{\prime}(1, w, z) & =\frac{U(w z)}{w} E_{l}(1, w, z) \\
& =\frac{1}{w} \frac{T(w z)^{l+1}-T(w z)^{l+2} / 2}{1-T(w z)} e^{U(w z) / w} .
\end{aligned}
$$

The factor $w^{-1}$ contributes a factor of $n / \lambda$ to the corresponding function $e_{l, n}(\lambda)$, according to $(3.13)$, hence we have

$$
e_{l, n}(\lambda)=\frac{n}{\lambda}\left(s_{l+1, n}(\lambda)-\frac{s_{l+2, n}(\lambda)}{2}\right) .
$$

The integral $\int_{1}^{\infty} s_{l, n}(\lambda) d \lambda / \lambda$ is of order $n^{-1 / 6}$, by the results of Section 4 , and we have in fact

$$
\int_{0}^{\infty} s_{l, n}(\lambda) \frac{d \lambda}{\lambda}=\frac{1}{2} \int_{0}^{1} \frac{\lambda^{l-2}}{\sqrt{1-\lambda}} d \lambda+O\left(n^{-1 / 6}\right) .
$$

Therefore the waiting time has a simple relation to cycle length probabilities: 
Corollary 3. The expected number of edges when an evolving graph obtains its first cycle is $\frac{1}{3} n+O\left(n^{5 / 6}\right)$, in the uniform model. It is $\frac{1}{2}\left(1-\hat{p}_{3}\right) n+O\left(n^{5 / 6}\right)$ in the permutation model, where $\hat{p}_{3}$ is the constant in (5.13).

Proof. Take $l=1$ and $l=3$ in (7.11), getting $n\left(P_{\geq 1, n}-\frac{1}{2} P_{\geq 2, n}\right)$ or $n\left(\widehat{P}_{\geq 3, n}-\frac{1}{2} \widehat{P}_{\geq 4, n}\right)+$ $O\left(n^{5 / 6}\right)$ as the expected values of $n-m$.

The variance can be shown, similarly, to have the respective values

$$
\left(\frac{31}{45} n^{2}, \frac{2+3 \hat{p}_{3}-\hat{p}_{3}^{2}-\hat{p}_{4}}{4} n^{2}\right)+O\left(n^{11 / 6}\right)
$$

We will examine another way to compute the expected waiting time in Section 10 below.

Finally, let us investigate the number of vertices that remain isolated when the first cycle appears. The relevant trivariate generating function is

$$
F_{l}(\zeta, w, z)=\frac{T(w, z)^{l}}{1-T(w, z)} e^{(U(w z)-w z+\zeta w z) / w},
$$

since we put the $\zeta$ marker on the unordered components of size 1 . In this case we find

$$
F_{l}^{\prime}(1, w, z)=z S^{l}(w, z)=w^{-1} T(w z) e^{-T(w z)} S_{l}(w, z) .
$$

Corollary 4. The expected number of isolated vertices when the first cycle appears in an evolving graph is

$$
\frac{n}{2} \int_{0}^{1} \frac{e^{-\lambda} d \lambda}{\sqrt{1-\lambda}}+O\left(n^{5 / 6}\right)
$$

in the uniform model, and

$$
\frac{n}{2} \int_{0}^{1} \frac{\lambda^{2} e^{-\lambda / 2+\lambda^{2} / 4}}{\sqrt{1-\lambda}} d \lambda+O\left(n^{5 / 6}\right)
$$

in the permutation model.

MACSYMA finds the integral in (7.16) to be $-e^{-1} \sqrt{\pi} i \operatorname{erf}(i)$; the coefficient of $n$ is therefore $\approx 0.53808$. The corresponding coefficient in $(7.17)$ is $\approx 0.42046$.

8. Cycle lengths versus edges. Let us now try to study the joint distribution of $l$ and $m$, the cycle length and the number of edges when the evolution procedure of Section 1 is applied to $n$ initially disconnected vertices. The corresponding probabilities will be called $P_{l, m, n}$ in the uniform model and $\widehat{P}_{l, m, n}$ in the permutation model.

We can express these probabilities directly from univariate generating functions, instead of using the more elaborate machinery of Theorem 1 . Let $C_{l, m, n}$ be the number of 
stopping configurations in which the process can stop with a cycle of length $l$ and with $m$ edges on $n$ vertices. Then there are $n-m$ components in the acyclic part, and we have

$$
C_{l, m, n}=\frac{n !}{(n-m) !}\left[z^{n}\right] T(z)^{l} U(z)^{n-m} .
$$

These numbers, incidentally, satisfy the recurrences

$$
\begin{aligned}
C_{l+1, m, n} & =\sum_{k \geq 1}\left(\begin{array}{l}
n \\
k
\end{array}\right) k^{k-1} C_{l, m-k, n-k} \\
(n-m) C_{l, m, n} & =\sum_{k \geq 1}\left(\begin{array}{l}
n \\
k
\end{array}\right) k^{k-2} C_{l, m+1-k, n-k} .
\end{aligned}
$$

The corresponding probabilities, as we have seen in Section 3, are

$$
\begin{aligned}
& P_{l, m, n}=\frac{2^{m-1}(m-1) !}{n^{2 m}} C_{l, m, n} \\
& \hat{P}_{l, m, n}=\frac{1}{2 m\left(\begin{array}{c}
\left(n^{2}-n\right) / 2 \\
m
\end{array}\right)} C_{l, m, n}=e^{m / n+m^{2} / n^{2}} P_{l, m, n}+O\left(n^{-1}\right) .
\end{aligned}
$$

Let us set $\lambda=2 m / n$. Erdös and Rényi [ER60] observed that an evolving graph on $n$ vertices changes its character dramatically when $m$ grows so that $\lambda$ passes the critical value $\lambda=1$. It turns out that, for sub-critical graphs $(\lambda<1)$, the quantity $P_{\geq 1, m, n}$ behaves very much like the function $s_{l, n}(\lambda)$ in Lemma 1 , except for a factor $2 / n$ (which corresponds to $d \lambda$ ):

Theorem 4. If $2 m / n=\lambda<1$ as $n \rightarrow \infty$, where $\delta \leq \lambda \leq 1-\delta$, we have

$$
P_{l, m, n}=\frac{\lambda^{l-1} \sqrt{1-\lambda}}{n}\left(1+O\left(\frac{l^{2}}{n \delta}\right)+O\left(\frac{1}{n^{1 / 2} \delta^{3 / 2}}\right)\right)
$$

uniformly in $\delta>0$ and $l \geq 1$.

Proof. We will apply the saddle point method to estimate the coefficient of $z^{n}$ in $T(z)^{l} U(z)^{n-m} /(1-T(z))$, thereby obtaining an asymptotic value of $C_{\geq 1, m, n}$. Again we replace $z$ by $z e^{-z}$ in order to obtain a simpler integral:

$$
\begin{aligned}
\frac{1}{2 \pi i} \oint \frac{T(z)^{l} U(z)^{n-m} d z}{(1-T(z)) z^{n+1}} & =\frac{1}{2 \pi i} \oint z^{l}\left(z-z^{2} / 2\right)^{n-m} e^{n z} \frac{d z}{z^{n}} \\
& =\frac{1}{2 \pi i} \oint z^{l} e^{n h(z)} d z,
\end{aligned}
$$


where in this case we have

$$
\begin{aligned}
h(z) & =z-\frac{\lambda}{2} \ln z+\left(1-\frac{1}{2} \lambda\right) \ln \left(1-\frac{1}{2} z\right) \\
h^{\prime}(z) & =1-\frac{\lambda}{2 z}-\frac{2-\lambda}{4-2 z} \\
h^{\prime \prime}(z) & =\frac{1}{2}\left(\frac{\lambda}{z^{2}}-\frac{2-\lambda}{(2-z)^{2}}\right)
\end{aligned}
$$

There are two saddle points, at $z=\lambda$ and $z=1$, just as we observed for a different function $h(z)$ in Section 4. (Is there an "obvious" reason why this should be true?) Again we have $h^{\prime \prime}(\lambda)>0$ and $h^{\prime \prime}(1)<0$, so we want to integrate on the circular path $|z|=\lambda$.

The real part of $h\left(\lambda e^{i \theta}\right)$ is now

$$
\lambda \cos \theta-\frac{1}{2} \lambda \ln \lambda+\frac{1}{2}\left(1-\frac{1}{2} \lambda\right) \ln L, \quad L=1+\frac{1}{4} \lambda^{2}-\lambda \cos \theta,
$$

and its second derivative is

$$
\frac{\lambda}{4}\left(\cos \theta\left(\frac{2-\lambda}{L}-4\right)-\frac{\lambda(2-\lambda) \sin ^{2} \theta}{L^{2}}\right) .
$$

This is negative when $\cos \theta \geq 0$, because

$$
\frac{2-\lambda}{L}-4 \leq \frac{2-\lambda}{(1-\lambda / 2)^{2}}-4=-4\left(\frac{1-\lambda}{2-\lambda}\right) \text {. }
$$

Therefore we can restrict attention once again to the neighborhood of $\theta=0$, and the result is

$$
\begin{aligned}
C_{\geq l, m, n}= & \frac{n !}{(n-m) !} \frac{1}{2 \pi i} \oint z^{l} e^{n h(z)} d z \\
= & \frac{n !}{(n-m) !} \frac{\lambda^{l} e^{n h(\lambda)}}{\sqrt{2 \pi n(1-\lambda)}} \sqrt{\frac{n-m}{m}} \\
& \quad \times\left(1+O\left(\frac{l^{2}}{n \lambda(1-\lambda)}\right)+O\left(\frac{1}{n^{1 / 2} \lambda^{3 / 2}(1-\lambda)^{3 / 2}}\right)\right) \\
= & \frac{\lambda^{l}}{\sqrt{1-\lambda}} \frac{n ! e^{n}}{\sqrt{2 \pi n} n^{n-2 m}} \frac{\sqrt{2 \pi(n-m)}}{(n-m) !} \frac{(n-m)^{n-m}}{e^{n-m}} \frac{e^{m} 2^{-m}}{\sqrt{2 \pi m} m^{m}} \\
& \left.\quad \times\left(1+O\left(l^{2} n^{-1} \delta^{-1}\right)\right)+O\left(n^{-1 / 2} \delta^{-3 / 2}\right)\right) \\
= & \frac{\lambda^{l}}{\sqrt{1-\lambda}} \frac{n^{2 m}}{m ! 2^{m}}\left(1+O\left(l^{2} n^{-1} \delta^{-1}\right)+O\left(n^{-1 / 2} \delta^{-3 / 2}\right)\right) .
\end{aligned}
$$


Now we can use (8.4) to conclude that

$$
P_{\geq l, m, n} \sim \frac{\lambda^{l-1}}{\sqrt{1-\lambda n}}
$$

as desired.

Theorem 4 gives us the promised "physical" interpretation of the parameter $\lambda$ in the machinery of Theorem 1: The running time $m$ of the random process is represented by $\frac{1}{2} \lambda n$, at least when $\lambda<1$. Thus, Figure 2 shows the approximate distribution of running times in the uniform model, when $n=20$ and $n=40$. A similar statement holds for the permutation model; but in that case we should consider the graph of $s_{3, n}(\lambda) e^{\lambda / 2+\lambda^{2} / 4}$ instead of $s_{1, n}(\lambda)$, because of (3.14) and (8.5).

It is interesting to note that, for fixed ratio $\lambda=2 m / n<1$ and for varying $l \ll \sqrt{n}$, the distribution of cycle lengths over all graphs whose first cycle occurs at time $m$ is approximately geometric in $l$, with parameter $\lambda$, except for a normalization factor.

If we set $l=3$ in (8.13) and apply (8.5), we get

$\operatorname{Pr}$ (Graph with $n$ vertices and $m$ edges has no cycle)

$$
\begin{aligned}
=\sum_{\mu>m} \hat{P}_{\geq 3, \mu, n} & \sim \frac{1}{2} \int_{\lambda}^{1} \frac{\lambda^{2}}{\sqrt{1-\lambda}} e^{\lambda / 2+\lambda^{2} / 4} d \lambda \\
& =\sqrt{1-\lambda} e^{\lambda / 2+\lambda^{2} / 4},
\end{aligned}
$$

if $\lim _{n \rightarrow \infty} 2 m / n=\lambda<1$, a classic result of Erdös and Rényi [ER60, Theorem $5 \mathrm{~b}$ ].

The situation changes when $m>\frac{1}{2} n$; in this "supercritical" case the ratio $2 m / n$ no longer represents the parameter $\lambda$ in Theorem 1 and Figure 2. (We might expect the relationship to break down when $\lambda$ is large, because the evolution process always stops with $m \leq n$; the $\lambda$ of Theorem 1 and Figure 2 is a continuous parameter that defines a positive but exponentially small function as $\lambda \rightarrow \infty$.) We can use the method of Theorem 3 when $\lambda>1$, integrating on the circle $|z|=1$, to deduce that

$$
P_{\geq l, m, n} \sim \frac{e^{-n(\lambda-1)}}{\lambda n \sqrt{\lambda-1}} \frac{\lambda^{m+1 / 2}}{(2-\lambda)^{n-m+1 / 2}}, \quad 1+\delta \leq \lambda \leq 2-\delta .
$$

(Compare with (4.18).) The probability $P_{l, m, n}$ is obtained if we insert the factor $(1-z)$ into the contour integrand; this introduces the factor $\frac{1}{2} \theta^{2}$ at the saddle point $\theta=0$, and the result is

$$
P_{l, m, n} \sim \frac{1}{2 n(\lambda-1)} P_{\geq l, m, n}=\frac{1}{4 m-2 n} P_{\geq l, m, n}, \quad 1+\delta \leq \lambda \leq 2-\delta .
$$


The method of Theorem 1 seems preferable to working directly with the actual probabilities $P_{l, m, n}$ for $m \geq \frac{1}{2} n$, because $s_{l, n}(\lambda)$ is a "smooth" function of $\lambda$ by which we can use uniform methods like Lemma 3 to span the critical region near $\lambda=1$.

9. Bicyclic components. Let's turn now to a related problem that can be handled with similar techniques. Instead of stopping the random graph or multigraph process when a cycle appears, let's keep it running until the first time there is a bicyclic componenta component with more than one cycle. If the first such component contains $j$ vertices it will have $j+1$ edges. The solution to this problem sheds more light on the generatingfunction-based techniques we have been discussing.

As before, we begin by defining and enumerating all of the stopping configurations in which our random process might terminate. The first bicyclic component can arise in one of two ways: Either (1) the final edge lies entirely within a component that was already unicyclic (a component that already contained a cycle), or (2) the final edge joins two different unicyclic components.

Our experiences so far suggest that we ought to look first at the uniform model, in which each step selects from $n^{2}$ ordered pairs $\langle x, y\rangle$ at random, since the uniform model tends to give formulas that are simpler than the ones arising in the permutation model.

The generating function for unicyclic components on $n$ labeled vertices turns out to be

$$
V(z)=\frac{1}{2} \ln \frac{1}{1-T(z)}=\frac{T(z)}{2}+\frac{T(z)^{2}}{4}+\frac{T(z)^{3}}{6}+\cdots .
$$

Here's why: Every cycle of length $l \geq 3$ corresponds to $2 l$ sequences of $l$ rooted trees, because we can list the trees of the cycle by starting at $l$ different places and we can traverse the cycle in two directions. Cycles of length $l<3$ have the forms $\langle x, x\rangle$ or $\langle x, y\rangle\langle x, y\rangle$; we will want to divide by $2 l$ in these cases also, because of the weighting. function $2^{m-1}(m-1)$ ! that will be applied later. (This weighting function assumes that a given multiset of $m$ edges containing no bicyclic components can arise in $2^{m-1}(m-1)$ ! ways as a sequence $\left\langle x_{1}, y_{1}\right\rangle \ldots\left\langle x_{m-1}, y_{m-1}\right\rangle$ of ordered pairs; but the actual number of ways is $2^{m-1-k}(m-1)$ !, where $k$ is the number of 1-cycles and 2-cycles, so we want to introduce a factor of $\frac{1}{2}$ for every such cycle.)

In case (1) the stopping configuration consists of a unicyclic component together with two special vertices $\langle x, y\rangle$ in that component, plus a set of any number of additional acyclic or unicyclic components. In case (2) the stopping configuration consists of an ordered pair of unicyclic components together with a vertex $x$ in the first and a vertex $y$ in the second, plus a set of additional acyclic or unicyclic components as before.

Let $\vartheta=z \frac{d}{d z}$ be the operator that multiplies the coefficient of $z^{n}$ by $n$. Then the egf for stopping configurations in case (1) is $\left(\vartheta^{2} V(z)\right) \exp (U(z)+V(z))$, and in case (2) it is 
$(\vartheta V(z))^{2} \exp (U(z)+V(z))$. (The operator $\vartheta$ selects a vertex, and $U(z)+V(z)$ enumerates acyclic and unicyclic components.) Using (7.6) we have

$$
\begin{aligned}
\vartheta V(z) & =\frac{1}{2} T(z) /(1-T(z))^{2} \\
\vartheta^{2} V(z) & =\frac{1}{2} T(z)(1+T(z)) /(1-T(z))^{4}
\end{aligned}
$$

Therefore the overall egf for stopping configurations comes to

$$
\left(\vartheta^{2} V(z)+(\vartheta V(z))^{2}\right) e^{U(z)+V(z)}=\frac{T(z)(2+3 T(z))}{4(1-T(z))^{9 / 2}} e^{U(z)}
$$

this is only slightly more complex than formula (2.7), the analogous egf for stopping configurations in the first cycle problem.

Once again we need to work with bgf's, so that we have access to the number of edges. The appropriate bivariate generating function for stopping configurations in the uniform model is easily deduced from our derivation of (9.4): We have

$$
S(w, z)=\frac{w T(w z)(2+3 T(w z))}{4(1-T(w z))^{9 / 2}} e^{U(w z) / w} .
$$

And as in Section 3, we can state that $S(w, z)$ expands to the sum $\sum_{m, n} s_{m, n} w^{m} z^{n} / n !$, where $2^{m-1}(m-1) ! s_{m, n} / n^{2 m}$ is the probability that the process stops when the $m$ th edge is introduced.

As a check, let's look at the coefficients for small $n$ :

$$
S(w, z)=\frac{w^{2}}{2} z+\frac{w^{2}+7 w^{3}}{2} z^{2}+\frac{4 w^{2}+60 w^{3}+261 w^{4}}{16} z^{3}+\cdots .
$$

When $n=3$, the respective probabilities that we stop at time $m=2,3,4$ are

$$
\frac{2^{1} \cdot 1 ! \cdot 3 ! \cdot 4}{3^{4} \cdot 16}=\frac{1}{27}, \quad \frac{2^{2} \cdot 2 ! \cdot 3 ! \cdot 60}{3^{6} \cdot 16}=\frac{20}{81}, \quad \frac{2^{3} \cdot 3 ! \cdot 3 ! \cdot 261}{3^{8} \cdot 16}=\frac{58}{81},
$$

and these sum to 1 as they should. In general, we have

$$
\Phi_{n} S=1, \quad \text { for all } n \geq 1 ;
$$

the operator $\Phi_{n}$ of Section 3 applies to the bicyclic problem as well as to the unicyclic problem, and we can use the simplifications of Theorem 1 just as we did before.

Now let's turn to the permutation model, in which cycles of lengths 1 and 2 are forbidden. The appropriate egf for cycles is therefore

$$
\widehat{V}(z)=V(z)-\frac{T(z)}{2}-\frac{T(z)^{2}}{4}=\frac{1}{2} \ln \frac{1}{1-T(z)}-\frac{T(z)}{2}-\frac{T(z)^{2}}{4},
$$


a formula noted by Wright [Wr77]. The egf for stopping configurations in case (2) is $(\vartheta \widehat{V}(z))^{2} \exp (U(z)+\widehat{V}(z))$, because we choose $x$ and $y$ in distinct components as before. But in case (1) the number of ordered pairs $\langle x, y\rangle$ is twice the number of edges not already present in the unicyclic component, so the appropriate egf for case (1) is

$$
\left(\vartheta^{2}-3 \vartheta\right) V(z) \exp (U(z)+\widehat{V}(z)) \text {. }
$$

Adding these cases together and introducing $w$ as before gives us the bgf for stopping configurations in the permutation model:

$$
\hat{S}(w, z)=w \frac{T(w z)^{4}\left(10-6 T(w z)+T(w z)^{2}\right)}{4(1-T(w z))^{9 / 2}} \exp \left(\frac{U(w z)}{w}-\frac{T(w z)}{2}-\frac{T(w z)^{2}}{4}\right) .
$$

If we write $\hat{S}(w, z)=\sum_{m, n} \hat{s}_{m, n} w^{m} z^{n} / n !$, then $\hat{s}_{m, n} / 2 m\left(\begin{array}{c}n(n-1) / 2 \\ m\end{array}\right)$ is the probability that the first bicyclic component arises in the permutation model when the $m$ th edge appears. The coefficients for small $n$ are

$$
\hat{S}(w, z)=\frac{5 w^{5}}{2} z^{4}+\frac{5 w^{5}+37 w^{6}}{2} z^{5}+\frac{5 w^{5}+79 w^{6}+367 w^{7}}{4} z^{6}+\cdots
$$

thus when $n=6$, the process stops at time $m=5,6,7$ with respective probabilities $\frac{30}{1001}$, $\frac{237}{1001}, \frac{734}{1001}$. We have

$$
\Psi_{n} \hat{S}=1, \quad \text { for all } n \geq 4,
$$

where $\Psi_{n}$ is the operator of Section 3. Notice that the coefficient of $z^{3}$ in $\hat{S}(w, z)$ is zero; a graph on 3 vertices never has more than one cycle, so we shouldn't look for bicyclic components in the permutation model unless $n \geq 4$. But when $n \geq 4$, we obtain a bicyclic component after at most $n+1$ edges have been added.

What is the size of the first bicyclic component? In the uniform model, the generating function

$$
\frac{w T(\zeta w z)(2+3 T(\zeta w z))}{4(1-T(\zeta w z))^{4}} \frac{e^{U(w z) / w}}{(1-T(w z))^{1 / 2}}
$$

puts $\zeta^{j}$ into each stopping configuration whose bicyclic component contains $j$ vertices. After differentiating with respect to $\zeta$ and setting $\zeta=1$, we obtain an expression for the expected bicyclic component size:

$$
\Phi_{n} \frac{2 w T(w z)\left(1+6 T(w z)+3 T(w z)^{2}\right)}{(1-T(w z))^{13 / 2}} e^{U(w z) / w} .
$$

A similar formula, with the same denominator $(1-T(w z))^{13 / 2}$, applies to the permutation model. If the factor $w$ weren't present, we would have a generating function of the form (7.3), with $k=\frac{13}{2}$; the $\Phi_{n}$ operator would then produce a result of order $n^{(2 k-3) / 6}=n^{5 / 3}$. The factor $w$ changes the integrand by $\lambda / n$, and $\lambda \approx 1$ in the region where the integral becomes unbounded; hence the $w$ essentially divides by $n$, and we can state the following result: 
Corollary 5. The expected size of the first bicyclic component in an evolving graph is of order $n^{2 / 3}$. The standard deviation is also of order $n^{2 / 3}$.

Corollary 6. The expected total length of the cycles in unicyclic components, at the moment when the first bicyclic component appears, is proportional to $n^{1 / 3}$.

Proof. Replace $(1-T(w z))^{1 / 2}$ by $(1-\zeta T(w z))^{1 / 2}$ in the denominator of $S(w, z)$, differentiate with respect to $\zeta$, and set $\zeta=1$.

We can find the expected waiting time $m$ by using the trick (7.9) that led to Corollary 3. In this case $n+1-m$ is the number of acyclic components, so the expected value of $n+1-m$ is

$$
\Phi_{n}\left(\frac{U(w z)}{w} S(w, z)\right) \quad \text { or } \quad \Psi_{n}\left(\frac{U(w z)}{w} \hat{S}(w, z)\right),
$$

depending on the model. In both cases the multiplication by $U(w z)=T(w z)-T(w z)^{2} / 2$ yields a numerator polynomial in $T(w z)$ whose value $\bmod (1-T(w z))$ is half what it was before. Since $\Phi_{n} S(w, z)=\Psi_{n} \hat{S}(w, z)=1$, and since division by $w$ contributes a factor of $n$, the waiting time must be asymptotically $\frac{1}{2} n$.

10. Waiting times revisited. When our goal is to find the average value of $m$, we can use another method based on generating functions for "going configurations" instead of stopping configurations. Namely, if $f_{m n}$ is the number of graphs with $n$ vertices and $m$ edges such that the random process is not stopped, we can use this information to calculate the probability that the process is still going after $m$ steps. The sum of these probabilities, over all $m$, is the expected waiting time.

In the first cycle problem, a going configuration is simply a forest (a collection of edges with no cycles); hence the bgf for going configurations is simply

$$
F(w, z)=\sum_{m, n} f_{m, n} w^{m} \frac{z^{n}}{n !}=e^{U(w z) / w}
$$

Each going configuration occurs with probability $2^{m} m ! / n^{2 m}$ in the uniform model, so the expected waiting time for a graph with $n$ vertices is $\sum_{m} 2^{m} m ! f_{m, n} / n^{2 m}$. The operator $\Phi_{n}$ of Section 3 computes $\sum_{m} 2^{m-1}(m-1) ! f_{m n} / n^{2 m}$, so it's almost what we want. We can obtain the desired operator for going configurations by first multiplying by $w$, getting the bgf $\sum_{n} f_{m, n} w^{m+1} z^{n} / n ! ;$ then applying $\Phi_{n}$ to get $\sum 2^{m} m ! f_{m, n} / n^{2 m+2}$; then multiplying by $n^{2}$. In other words, the expected waiting time in the uniform model is

$$
n^{2} \Phi_{n} w F(w, z)
$$


Alternatively, we can obtain the desired operator by first differentiating with respect to $w$ (getting $\sum_{m} m f_{m, n} w^{m-1} z^{n} / n !$ ), then multiplying by $2 w$ and applying $\Phi_{n}$. In other words,

$$
2 \Phi_{n} w \frac{\partial}{\partial w} F(w, z)+f_{0, n}
$$

gives the same result as (10.2). (We must add in the term $f_{0, n}$, which is annihilated by differentiation.) Indeed, we have the operator identity

$$
n^{2} \Phi_{n} w=2 \Phi_{n} w \frac{\partial}{\partial w}
$$

valid when applied to any bgf with $F(0, z)=0$. Since $w \frac{\partial}{\partial w}=\frac{\partial}{\partial w} w-1$, we can rewrite (10.4) as follows:

$$
\frac{n^{2}}{2} \Phi_{n}=\Phi_{n}\left(\frac{\partial}{\partial w}-w^{-1}\right)
$$

Comparing (10.3) with our formula (7.10) for the average of $n-m$ yields the interesting identity

$$
\Phi_{n}\left(\left(\frac{1}{1-T}-1-T+3 T^{2}\right) \frac{e^{U(w z) / w}}{2 w}\right)=n-1, \quad T \equiv T(w z),
$$

which does not obviously follow from (10.5) and any other identities that we know. It may be possible to find a family of formulas such as this, allowing us to deduce nonobvious relations between different statistics on random graphs.

In the permutation model, the relevant formula for expected waiting time is

$$
2 \Psi_{n} w \frac{\partial}{\partial w} F(w, z)+f_{0, n}
$$

as in (10.3). There is apparently no simple analog of (10.2), although we can derive a formula that is somewhat like (10.5):

$$
\left(\frac{n(n-1)}{2}+1\right) \Psi_{n}=\Psi_{n}\left((1+w) \frac{\partial}{\partial w}-w^{-1}\right)
$$

The identity analogous to (10.6) is

$$
\Psi_{n}\left(\left(\frac{1}{1-T}-1-T+T^{2}-T^{3}+T^{4}\right) \frac{e^{U(w z) / w}}{2 w}\right)=n-1, \quad T \equiv T(w z),
$$

valid for $n \geq 3$.

The bgf for going configurations in the problem of bicyclic components is

$$
e^{U(w z) / w+V(w z)} \quad \text { or } \quad e^{U(w z) / w+\widehat{V}(w z)},
$$


depending on the model, because the process keeps going if and only if the graph components are acyclic or unicyclic. The formulas in (9.12) now lead, via (10.3) and (10.7), to further identities like (10.6) and (10.9):

$$
\begin{gathered}
\Phi_{n}\left(\left(\frac{T^{2}}{w}-\frac{3}{8}+\frac{3-4 T+6 T^{2}}{8(1-T)^{4}}\right) \frac{e^{U(w z) / w}}{\sqrt{1-T}}\right)=n \\
\Psi_{n}\left(\left(\frac{T^{2}}{w}+\frac{1+4 T^{3}+T^{4}}{8}+\frac{-1+4 T-6 T^{2}+8 T^{3}}{8(1-T)^{4}}\right) \frac{e^{U(w z) / w-T / 2-T^{2} / 4}}{\sqrt{1-T}}\right)=n .
\end{gathered}
$$

(Again $T$ stands for $T(w z)$, and the identity for $\Psi_{n}$ holds only when $n \geq 3$.) Is there a simple combinatorial or algebraic principle that accounts for amazing formulas like this?

We have observed in Section 9 that the waiting time for the first bicyclic component is approximately $\frac{1}{2} n$; thus, the graph tends to become bicyclic when $m$ passes the critical value where random graphs rapidly gain a complex structure. It is interesting to look more closely at this transitional phase, by studying the probability that there is not yet a bicyclic component when $m \approx \frac{1}{2} n$. For this purpose we can combine the ideas used to prove Lemma 3 in Section 4 and Theorem 4 in Section 8.

Theorem 5. Let $\lambda=2 m / n=e^{-\mu \nu}$, where $\nu=n^{-1 / 3}$. Then the probability that a random graph with $n$ vertices and $m$ edges has no bicyclic component is

$$
\pi_{m, n}=\frac{1}{\sqrt{2 \pi} i} \int_{1-i \infty}^{1+i \infty} s^{1 / 2} e^{(\mu+2 s)(\mu-s)^{2} / 6} d s+O\left(n^{-1 / 4}\right),
$$

uniformly for $|\mu| \leq n^{1 / 12}$.

Proof. We have

$$
\pi_{m, n}=\frac{n !}{(n-m) !\left(\begin{array}{l}
N \\
m
\end{array}\right)}\left[z^{n}\right] U(z)^{n-m} e^{\widehat{V}(z)}
$$

where $\widehat{V}(z)$ is defined in (9.7) and $N=\left(\begin{array}{c}n \\ 2\end{array}\right)$. Let $h(z)$ be the function defined in (8.8); then, as in that derivation,

$$
\left[z^{n}\right] U(z)^{n-m} e^{\widehat{V}(z)}=\frac{1}{2 \pi i} \oint \sqrt{1-z} e^{-z / 2-z^{2} / 4} e^{n h(z)} \frac{d z}{z}
$$

Let $z=e^{(i t-\alpha) \nu}$. A tedious but straightforward calculation shows that (10.15) equals

$$
\frac{n^{-1 / 2} e^{-3 / 4-\mu^{3} / 6+n}}{2 \pi 2^{n-m} i} \int_{1-i \infty}^{1+i \infty} s^{1 / 2} e^{P(\mu, s)} d s(1+O(\beta(\alpha, n) \nu))
$$


if we argue as in Lemma 3. Furthermore the quantity $n ! /(n-m) !\left(\begin{array}{l}N \\ m\end{array}\right)$ in $(10.14)$ can be shown to equal

$$
\sqrt{2 \pi n} e^{3 / 4+\mu^{3} / 6-n} 2^{n-m}\left(1+O\left(n^{-1 / 3} \ln n\right)\right) .
$$

Multiplying (10.16) by (10.17) yields (10.13).

When $\mu \rightarrow-\infty$, the value of $\pi_{m, n}$ in (10.13) is exponentially small; in fact it is $O\left(e^{\mu^{3} / 6-\mu / 2}\right)$, because

$$
\Re P(\mu, 1+i y)=\frac{(\mu+2)(\mu-1)^{2}-(6-3 \mu) y^{2}}{6}
$$

On the other hand, when $\mu \rightarrow+\infty$ we can prove that $\pi_{m, n}=1+O\left(\mu^{-3}\right)$, by integrating on the path $s=\mu+i y / \sqrt{\mu}$ for $-\infty<y<\infty$. For we have $P(\mu, \mu+i y / \sqrt{\mu})=-y^{2} / 2-$ $i y^{3} /\left(3 \mu^{3 / 2}\right)$; the integral can be restricted to $|y| \leq \ln n$, in which range the integrand is $e^{-y^{2} / 2} i d y$ times $1+\frac{1}{2} i y \mu^{-3 / 2}-\frac{1}{2} i y^{3} \mu^{-3 / 2}+O\left(\left(y^{2}+y^{6}\right) \mu^{-3}\right)$. Therefore the random graph process almost always keeps going without bicyclic components until the number of edges is on the order of $\frac{1}{2} n e^{-\mu \nu} \approx \frac{1}{2} n-\frac{1}{2} \mu n^{2 / 3}$. If we take $M$ large enough, the probability is $\geq 1-\epsilon$ that the first bicyclic component occurs when $\frac{1}{2} n-M n^{2 / 3} \leq m \leq \frac{1}{2} n+M n^{2 / 3}$. Informally we can say that the graph almost certainly becomes bicyclic when the number of edges is $\frac{1}{2} n+\Theta\left(n^{2 / 3}\right)$.

When $\lambda$ is strictly less than 1 , say $\lambda \leq 1-\delta$, we can show that $\pi_{m, n}=1-O\left(n^{-1} \delta^{-1}\right)-$ $O\left(n^{-1 / 2} \delta^{-3 / 2}\right)$ by integrating on the contour $z=\lambda e^{i \theta}$ as in the proof of Theorem 4. (See [ER60, Theorem 5e].) We can now sharpen the result of Erdős and Renyi stated in (8.14):

Corollary 7. Let $L$ be a set of positive integers, and say that an $L$-cycle is a cycle whose length is in $L$. Then

$\operatorname{Pr}$ (Graph or multigraph with $n$ vertices and $m$ edges has no $L$-cycle)

$$
=\sqrt{1-\lambda} \exp \left(\sum_{\substack{l \geq 1 \\ l \in \mathbb{E} L}} \frac{\lambda^{l}}{2 l}\right)+O\left(n^{-1 / 2}\right)
$$

if $\lim _{n \rightarrow \infty} 2 m / n=\lambda<1$.

(This result applies to graphs as well as multigraphs; we assume that $1 \notin L$ and $2 \notin L$ when we are considering graphs. A multigraph can have self loops (1-cycles) and/or repeated edges (2-cycles), but a graph cannot.)

Proof. The multigraph either contains a bicyclic component or it doesn't. The first case occurs with probability $O\left(n^{-1 / 2}\right)$. In the second case we want the probability of a "going 
configuration" that consists entirely of acyclic components and unicyclic components whose cycle lengths are $\notin L$. The number of such configurations is

$$
\left[z^{n}\right] U(z)^{n-m} \exp \left(\sum_{\substack{1 \geq 1 \\ l \notin L}} \frac{T(z)^{l}}{2 l}\right)
$$

so we are able to complete the estimates by repeating almost verbatim the argument of Theorem 4.

If we set $L=\{1,2\}$ in $(10.18)$, we get the asymptotic probability that a random multigraph is a graph, namely $e^{-\lambda / 2-\lambda^{2} / 4}$. If we set $L=\{3,5,7,9, \ldots\}$, we get the asymptotic probability that a random graph is 2 -colorable, namely

$$
\sqrt{1-\lambda} \exp \left(\frac{1}{2} \lambda-\frac{1}{4} \ln \left(1-\lambda^{2}\right)\right)=\left(\frac{1-\lambda}{1+\lambda}\right)^{1 / 4} e^{\lambda / 2}
$$

Otherwise [ER60, $§ 10]$, such a graph is almost surely 3 -colorable when $\lambda<1$.

Choosing $L=\{k+1, k+2, \ldots\}$ in (10.18) gives the limiting distribution of the longest cycle in a random graph: All cycle lengths are $\leq k$ with probability

$$
\sqrt{1-\lambda} \exp \left(\sum_{l=1}^{k} \frac{\lambda^{l}}{2 l}\right)+O\left(n^{-1 / 2}\right)
$$

(An analogous result has been derived by Pittel [Pi88, Theorem 1], for random graphs in which each edge occurs independently with probability $\lambda / n$.)

Erdős and Renyi [ER60, $\S 8$ ] stated that, if $r$ is any real number, the probability that a graph with $n$ vertices and $\frac{1}{2} n+r n^{1 / 2}$ edges is non-planar "has a positive lower limit, but we cannot calculate its value. It may even be 1 , though this seems unlikely." We can now show that this probability is definitely less than 1. Indeed, a graph with $n$ vertices and $\frac{1}{2} n+r n^{1 / 2}$ edges has $\mu \approx 2 r n^{-1 / 6}$ in the hypothesis of Theorem 5 , so the probability that it has no bicyclic component (and is therefore planar) approaches the limiting value stated for $\mu=0$ and $\alpha=1$. We can prove, in fact, that this limiting value $\pi_{n / 2, n}$ is rather large:

Corollary 8. The probability that a graph with $n$ vertices and $\frac{1}{2} n$ edges has no bicyclic component is $\sqrt{2 / 3}+O\left(n^{-1 / 3}\right)$.

Proof. The contour integral in (10.15) is particularly interesting when $\lambda=1$ because it has a three-legged saddle point. One way to evaluate it is to consider a path of the form $z=1+t e^{2 \pi i / 3} n^{-1 / 3}$ for $t \geq 0$; this accounts for half of (10.15), and the result turns out to be

$$
\frac{n^{-1 / 2} e^{n-3 / 4}}{2^{n / 2} \pi} \int_{0}^{\infty} \sqrt{t} e^{t^{3} / 3} d t\left(1+O\left(n^{-1 / 3}\right)\right)
$$


We will see in formula (A.8) below that this integral is $\frac{1}{3} \sqrt{3 \pi}$. The auxiliary coefficient $n ! /(n-m) !\left(\begin{array}{c}N \\ m\end{array}\right)$ is $\sqrt{2 \pi n} e^{3 / 4-n} 2^{n / 2}\left(1+O\left(n^{-1}\right)\right)$ when $m=\frac{1}{2} n$.

Erdös and Renyi [ER60] also remarked that a graph with $\frac{1}{2} n+\omega_{n} \sqrt{n}$ vertices has a cycle with any given number of diagonals, with probability $\rightarrow 1$ when $\omega_{n} \rightarrow+\infty$ and $n \rightarrow \infty$. However, we have just proved that this is not true when $\omega_{n}=n^{1 / 6}$. Therefore the claim that a graph with exactly $\frac{1}{2} n$ edges has positive probability of nonplanarity might also be false; an explicit proof or disproof would be desirable.

11. The first $k$ cycles. As a final example of the techniques we have been considering, let us study the distribution of the first $k$ cycles that appear in an evolving graph. We have seen in Section 10 that this problem is well-defined, at least asymptotically, because the first cycles in a sufficiently large graph will almost always occur in distinct components.

For simplicity let us once again consider the uniform model first. We will run the random multigraph process until there is either a bicyclic component or a set of $k$ unicyclic components, whichever occurs first. In the latter case we let $l_{1}, l_{2}, \ldots, l_{k}$ be the lengths of the first $k$ cycles, in order of appearance.

A stopping configuration in the non-bicyclic case will consist of a sequence of cycles of rooted trees, having respective lengths $\left(l_{1}, \ldots, l_{k-1}\right)$, together with a sequence of $l_{k}$ rooted trees, plus a set of any number of unrooted trees. A cycle of $l$ rooted trees has the egf $T(z)^{l} / 2 l$, as discussed in (9.1). Therefore if we form the multivariate generating function

$$
\begin{aligned}
S\left(x_{1}, \ldots, x_{k}, w, z\right) & =\sum_{l_{1}, \ldots, l_{k} \geq 1} \frac{T(w z)^{l_{1}}}{2 l_{1}} \ldots \frac{T(w z)^{l_{k-1}}}{2 l_{k-1}} T(w z)^{l_{k}} x_{1}^{l_{1}} \ldots x_{k}^{l_{k}} e^{U(w z) / w} \\
& =\left(\prod_{j=1}^{k-1}\left(\frac{1}{2} \ln \frac{1}{1-x_{j} T(w z)}\right)\right) \frac{x_{k} T(w z)}{1-x_{k} T(w z)} e^{U(w z) / w}
\end{aligned}
$$

the coefficient $n !\left[x_{1}^{l_{1}} \ldots x_{k}^{l_{k}} w^{m} z^{n}\right] S\left(x_{1}, \ldots, x_{k}, w, z\right)$ will be the number of stopping configurations with $m$ edges, $n$ vertices, and cycle lengths $\left(l_{1}, \ldots, l_{k}\right)$.

In order to convert these coefficients to probabilities, we need to consider how many of the $n$ sequences $\left\langle x_{1}, y_{1}\right\rangle \ldots\left\langle x_{m}, y_{m}\right\rangle$ of edges will yield a stopping configuration with parameters $m, n, l_{1}, \ldots, l_{k}$. For this we need a slight generalization of the argument at the beginning of Section 3; the appropriate factor is now not $2^{m-1}(m-1) ! / n^{2 m}$ but rather

$$
\frac{2^{m-1}(m-1) !}{n^{2 m}} \frac{l_{1}}{L_{1}} \frac{l_{2}}{L_{2}} \cdots \frac{l_{k-1}}{L_{k-1}}
$$

where

$$
L_{j}=l_{1}+\cdots+l_{j}
$$


The reason is that the $(m-1)$ ! permutations of the $m-1$ non-final edges are not all admissible. Exactly $l_{k-1} / L_{k-1}$ of them have the final edge of the $(k-1)$ st cycle occurring after all the edges of the first $k-2$ cycles; and $l_{k-2} / L_{k-2}$ of these have the final edge of the $(k-2)$ nd cycle occurring after all the edges of the first $k-3$; and so on.

The stopping configurations in the bicyclic case can be ignored, because we know that this case occurs with vanishing probability as $n \rightarrow \infty$; but we might as well describe the generating function, so that we can see how rapidly the probability approaches zero. We mimic the derivation of (9.4): Either $k \geq 2$ and there is a unicyclic component with two marked vertices, plus an additional set of acyclic and (at most $k-2$ ) unicyclic components; or $k \geq 3$ and there are two unicyclic components with marked vertices plus an additional set of acyclic and (at most $k-3$ ) unicyclic components. The egf is therefore

$$
\left(\vartheta^{2} V(z)\right) e^{U(z)} \sum_{j=0}^{k-2} \frac{V(z)^{j}}{j !}+(\vartheta V(z))^{2} e^{U(z)} \sum_{j=0}^{k-3} \frac{V(j)^{j}}{j !}
$$

Converting to' a bgf gives a formula like (9.5) except that it has the form

$$
\frac{w f_{k}(T(w z))}{(1-T(w z))^{4}} e^{U(w z) / w}
$$

where $f_{k}$ is a polynomial. By reasoning as we did after (9.11), we conclude that the $\Phi_{n}$ operator produces a result that is $O\left(n^{-1} n^{(8-3) / 6}\right)=O\left(n^{-1 / 6}\right)$, for every fixed $k$.

We can now determine the asymptotic probability that a given sequence of cycle lengths will appear:

Theorem 6. The probability that the random multigraph process produces the first $k$ cycles in distinct components with respective lengths $\left(l_{1}, l_{2}, \ldots, l_{k}\right)$ is

$$
\frac{2^{1-k}}{L_{1} L_{2} \ldots L_{k-1}} p_{L_{k}}+O\left(n^{-1 / 6}\right)
$$

for all fixed $l_{1}, \ldots, l_{k} \geq 1$, where $L_{j}$ is defined in (11.3) and $p_{l}$ is defined in (5.6). The same formula holds for the random graph process, if $p$ is replaced by $\hat{p}$ and if we require $l_{1} \ldots l_{k} \geq 3$.

Proof. The desired probability, according to (11.1), (11.2), and (3.1), is

$$
\begin{array}{r}
\Phi_{n}\left(\frac{l_{1}}{L_{1}} \frac{l_{2}}{L_{2}} \ldots \frac{l_{k-1}}{L_{k-1}}\left[x_{1}^{l_{1}} \ldots x_{k}^{l_{k}}\right] S\left(x_{1}, \ldots, x_{k}, w, z\right)\right) \\
=\frac{2^{1-k}}{L_{1} L_{2} \ldots L_{k-1}} \Phi_{n}\left(T(w z)^{L_{k}} e^{U(w z) / w}\right),
\end{array}
$$


plus $O\left(n^{-1 / 6}\right)$ for the probability of failure due to the early occurrence of a bicyclic component. And $\Phi_{n}\left(T(w z)^{l} e^{U(w z) / w}\right)$ is the probability that the first cycle has length $l$, computed in Theorem 2.

This proves (11.6) in the uniform model; the same ideas apply to the permutation model, with minor changes.

The probability distribution in Theorem 6 was first derived by Svante Jansen [Ja87], without the error term, in the case of random graphs. We can show that the sum of probabilities (11.6) over all $\left(l_{1}, \ldots, l_{k}\right)$ equals 1 , by using the identities

$$
\sum_{k=l+1}^{\infty} p_{k}=2 l_{p_{l}}, \quad \sum_{k=l+3}^{\infty} \hat{p}_{k}=2 l_{\hat{p}_{l}}
$$

already mentioned in (5.8) and (5.10). Notice that the asymptotic probability in the uniform model that the first $k$ cycles will all be loops of length 1 is $p_{k} / 2^{k-1}(k-1)$ ! = $1 /(3 \cdot 5 \cdot \ldots \cdot(2 k+1))$.

On intuitive grounds we expect the second cycle to be larger than the first, and the third should be larger yet, because the trees that yield cycles gradually get bigger. And indeed, this is true:

Theorem 7. The average length of the $k$ th cycle, for fixed $k$, is of order $n^{1 / 6}(\log n)^{k-1}$.

Proof. It suffices to give the proof for the uniform model, since the other model is similar.

The basic idea is to apply the identity

$$
\frac{z}{(1-z)^{2}}\left(\ln \frac{1}{1-z}\right)^{k-1}=(k-1) ! \sum_{l_{1}, \ldots, l_{k} \geq 1} \frac{l_{k} z^{L_{k}}}{L_{1} L_{2} \ldots L_{k-1}}
$$

which is readily verified by induction. The average value of $l_{k}$ is

$$
\sum_{l_{1}, \ldots, l_{k} \geq 1} l_{k} P\left(l_{1}, \ldots, l_{k}, n\right)
$$

where $P\left(l_{1}, \ldots, l_{k}, n\right)$ is the probability in (11.7); thus we want to apply $\Phi_{n}$ to the bgf

$$
\frac{1}{2^{k-1}(k-1) !} \frac{T}{(1-T)^{2}}\left(\ln \frac{1}{1-T}\right)^{k-1} e^{U(w z) / w}
$$

where $T \equiv T(w z)$. And it should be clear from the calculations in Sections 5 and 6 that the principal effect of each additional factor $\ln 1 /(1-T)$ is to multiply the inner integral by $\ln 1 /\left(1-e^{-\alpha \nu}\right)=\left(\frac{1}{3} \ln n+O(\ln (1+|\mu|))\left(1+O\left(n^{-1 / 3}\right)\right)\right.$. Therefore the result is $\Theta(\log n)^{k-1}$ times the result of Theorem 3 . 
In this proof we have defined the random variable $l_{k}$ to be zero if the first $k$ cycles are not well separated, i.e., if they do not fall in distinct components. This seems reasonable because the concept of $k$ th cycle becomes murky when many cycles are formed simultaneously.

A somewhat paradoxical situation arises if we ask for the conditional expected length of the first cycle, given that the first $k$ cycles appear in different components. For example, suppose $k=2$. Let $a_{n}$ be the unconditional expected length of the first cycle; let $b_{n}$ be the probability that the first two cycles are well separated; and let $c_{n} / b_{n}$ be the conditional expected length of the first cycle given that the first two cycles are well separated. Then we find

$$
\begin{aligned}
& a_{n}=\Phi_{n}\left(\frac{T}{(1-T)^{2}} e^{U(w z) / w}\right) \sim K n^{1 / 6} ; \\
& b_{n}=1-\Phi_{n}\left(\frac{w T(1+T)}{2(1-T)^{4}} e^{U(w z) / w}\right)=1-O\left(n^{-1 / 6}\right) ; \\
& c_{n}=\sum_{l_{1}, l_{2} \geq 1} l_{1} P\left(l_{1}, l_{2}, n\right)=\Phi_{n}\left(\frac{T^{2}}{2(1-T)^{2}} e^{U(w z) / w}\right),
\end{aligned}
$$

where $T \equiv T(w z)$. Since $T^{2} /(1-T)^{2}=T /(1-T)^{2}-T /(1-T)$, we have $c_{n}=\frac{1}{2}\left(a_{n}-1\right)$ exactly. Thus the expected value $c_{n} / b_{n}$ is asymptotically only half of $a_{n}$, although both quantities represent the expected length of the first cycle, and although we are conditioning on an event that almost surely occurs! The reason is that the distribution of first cycle lengths has a tail that decays very slowly; and cases when the first cycle is extremely long are much more likely to attract the second cycle into the same component.

Similarly, it can be shown that the conditional expected length of the the first cycle, given that the first $k$ cycles appear in separate components, is asymptotic to $2^{1-k} a_{n}$.

12. Concluding remarks. We have shown that a combination of generating functions and contour integration can resolve problems that apparently could not be treated successfully with the techniques that have previously been applied to random graphs. Many of the previous techniques, like the laws of large numbers, can be based on special cases of contour integration with the saddle point method; the approach in this paper may have succeeded primarily because we were free to use the saddle point method in a more general context.

It would be interesting to push the techniques further, for example by determining the asymptotic value of $L_{n}-K n^{1 / 6}$ when $L_{n}$ denotes the expected first cycle length. 
Appendix. Evaluation of integrals. Let us complete this discussion by studying the behavior of the integral in Lemma 3, equation (4.19), and by finding a numerical estimate for the constant $K$ in (6.8). This proves to be an interesting exercise in the theory of functions.

If $x$ is a real number, let us define

$$
\begin{aligned}
& f(x)=\int_{-\infty}^{\infty} \exp \left(-i t-x t^{2}+i t^{3} / 3\right) d t \\
& g(x)=\int_{-\infty}^{\infty} \exp \left(i x t+i t^{3} / 3\right) d t
\end{aligned}
$$

The motivation for $f(x)$ comes from the integral in (4.19), which reduces to a multiple of $f\left(\left(\alpha+\alpha^{-1}\right) / 2\right)$ under the substitions $s=\alpha-i t$ and $\mu=\alpha-\alpha^{-1}$. Since our main application of $f(x)$ has $x=\left(\alpha+\alpha^{-1}\right) / 2 \geq 1$, we can assume that $x \geq 1$ in $f(x)$. We can write

$$
f(x)=\int_{-\infty}^{\infty} e^{-x t^{2}} \cos \left(-t+t^{3} / 3\right) d t
$$

so $f(x)$ clearly converges for all $x>0$. We will prove that the related function $g(x)$ converges for all real $x$ (even though the integrand in its definition always has magnitude 1 ).

If $a$ is any positive number, we have

$$
\begin{aligned}
\left|\int_{R}^{R+i a} \exp \left(i x t+\frac{i t^{3}}{3}\right) d t\right| & \leq \int_{0}^{a}\left|\exp \left(i x(R+i t)+\frac{i(R+i t)^{3}}{3}\right)\right| d t \\
& =\int_{0}^{a} e^{-x t-R^{2} t+t^{3} / 3} d t \\
& \leq e^{a^{3 / 3+a|x|}} \int_{0}^{a} e^{-R^{2} t} d t=O\left(R^{-2}\right)
\end{aligned}
$$

a similar bound applies if we integrate from $-R$ to $-R+i a$. Hence we can shift the path of integration upward, without affecting the value or the convergence of the integral:

$$
g(x)=\int_{-\infty}^{\infty} \exp \left(i x(t+i a)+i(t+a i)^{3} / 3\right) d t, \quad a \geq 0 .
$$

There is now a term $-a t^{2}$ in the exponent, so $g(x)$ must indeed converge.

In particular, we have

$$
\begin{aligned}
g\left(a^{2}-1\right) & =\int_{-\infty}^{\infty} \exp \left(a-\frac{2}{3} a^{3}-i t-a t^{2}+\frac{1}{3} i t^{3}\right) d t \\
& =e^{a-2 a^{3} / 3} f(a) .
\end{aligned}
$$


Thus $f(x)=e^{2 x^{3 / 3-x}} g\left(x^{2}-1\right)$. When $x$ is large, we have $f(x)=\sqrt{2 \pi} x^{-1 / 2}+O\left(x^{-3 / 2}\right)$, hence $g\left(x^{2}-1\right)$ must be mighty small.

Another formula for $g(x)$ can be obtained by rotating the path of integration:

$$
\begin{aligned}
g(x) & =2 \Re \int_{0}^{\infty} \exp \left(i x t-i t^{3} / 3\right) d t \\
& =2 \Re\left(\zeta \int_{0}^{\infty} \exp \left(i x \zeta t+i \zeta^{3} t^{3} / 3\right) d t\right), \\
& =2 \Re\left(\zeta \int_{0}^{\infty} \exp \left(i x \zeta t-t^{3} / 3\right) d t\right),
\end{aligned}
$$

where

$$
\zeta=e^{\pi i / 6}=\frac{\sqrt{3}}{2}+\frac{1}{2} i .
$$

(The integral on the arc $R e^{i \theta}$ for $0 \leq \theta \leq \pi / 6$ is negligible for large $R$, because the magnitude of the integrand is $\exp \left(-\frac{1}{3} R^{3} \sin 3 \theta-x R \sin \theta\right)$.)

Equation (A.6) will be our key to evaluating $f(x)$ via $g(x)$, because we can expand $\exp (i x \zeta t)$ into a convergent power series in $t$. Then we can interchange summation and integration, evaluating the resulting integrals by using an analog of (4.14):

$$
\begin{aligned}
\int_{0}^{\infty} t^{k} e^{-t^{3} / a} d t & =\int_{0}^{\infty}(u a)^{k / 3} e^{-u} \frac{a d u}{3(u a)^{2 / 3}} \\
& =\frac{1}{3} a^{(k+1) / 3} \int_{0}^{\infty} u^{(k-2) / 3} e^{-u} d u \\
& =\frac{1}{3} a^{(k+1) / 3} \Gamma((k+1) / 3) .
\end{aligned}
$$

It follows that

$$
g(x)=\frac{2}{3^{2 / 3}} \Re\left(\sum_{k \geq 0} \zeta^{k+1} i^{k}(3 x)^{k} \frac{\Gamma((k+1) / 3)}{k !}\right) .
$$

The real part of $\zeta^{k+1} i^{k}$ is $\cos \left(\frac{2}{3} k+\frac{1}{6}\right) \pi$, which is respectively $\left(\frac{1}{2} \sqrt{3},-\frac{1}{2} \sqrt{3}, 0\right)$ when $k=$ $(0,1,2) \bmod 3 ;$ hence

$$
g(x)=3^{-1 / 6} \sum_{k \geq 0} \frac{\left(3^{1 / 3} x\right)^{3 k}}{(3 k+1) !}\left((3 k+1) \Gamma\left(k+\frac{1}{3}\right)-3^{1 / 3} x \Gamma\left(k+\frac{2}{3}\right)\right) .
$$

This series converges for all $x$; hence $g(z)$ and $f(z)$ are actually analytic functions in the entire complex plane.

We can write $(A .10)$ as a difference of two hypergeometric series of type ${ }_{0} F_{1}$ :

$$
g(x)=3^{-1 / 6} \Gamma\left(\frac{1}{3}\right) F\left(; \frac{2}{3} ; \frac{1}{9} x^{3}\right)-3^{1 / 6} x \Gamma\left(\frac{2}{3}\right) F\left(; \frac{4}{3} ; \frac{1}{9} x^{3}\right) .
$$




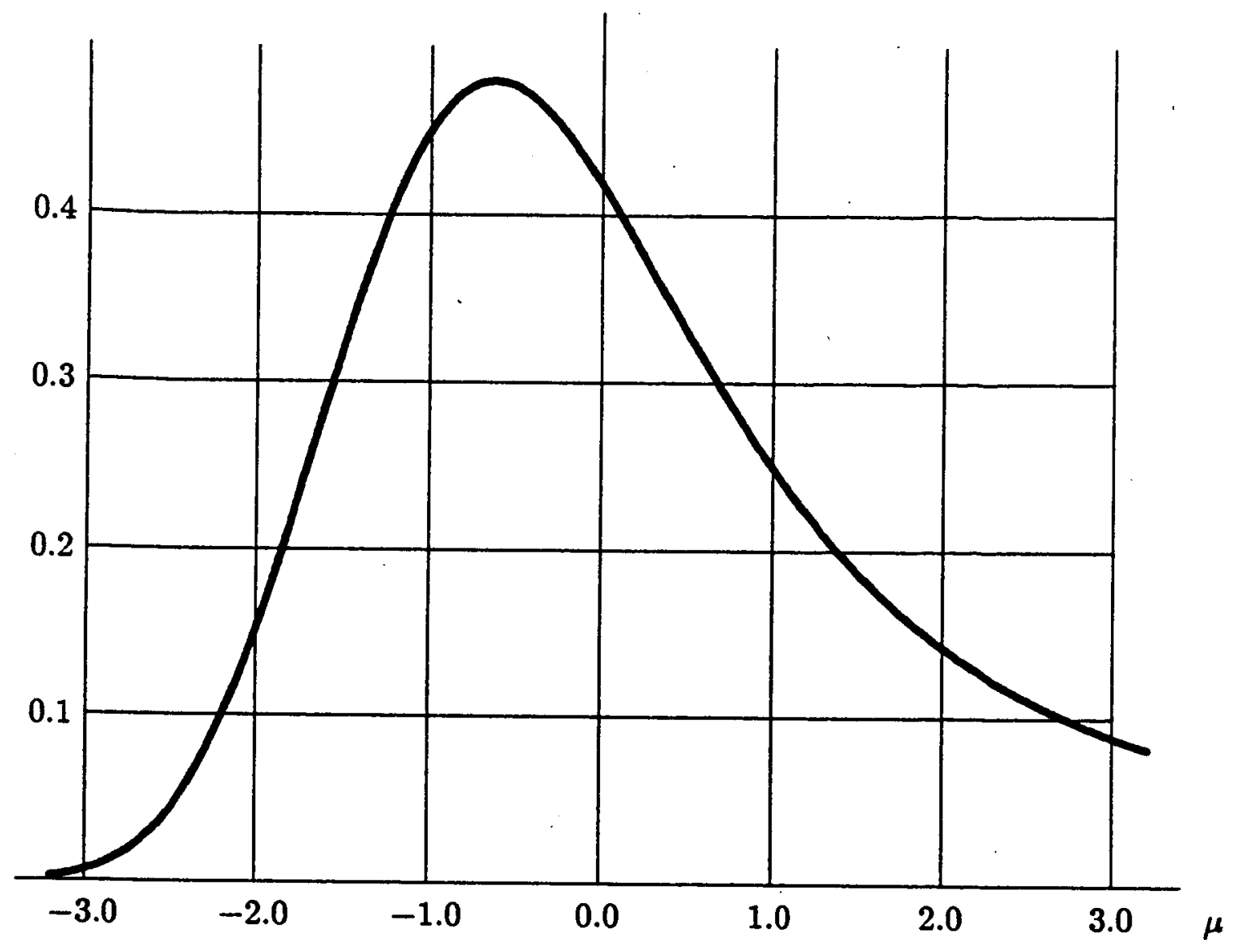

Figure 4. The function $K(\mu)$ whose integral yields the first-cycle constant $K$.

This representation allows us to deduce that $g(z)$ can be expressed as an Airy function, hence as a modified Bessel function of fractional order:

$$
g(z)=2 \pi \operatorname{Ai}(z)=\frac{2 z^{1 / 2}}{3^{1 / 2}} K_{1 / 3}\left(\frac{2}{3} z^{3 / 2}\right) .
$$

Equation (4.24) follows from the fact that $f(1)=e^{-1 / 3} g(0)$. In general, our derivation leads from (4.19) to the asymptotic formula

$$
s_{l, n}\left(e^{-\mu \nu}\right) \sim \frac{e^{\mu^{3} / 12} g\left(\mu^{2} / 4\right)}{\sqrt{8 \pi}} n^{1 / 6} .
$$

A somewhat different approach appears to be necessary if we want to evaluate the constant $K$ numerically. Let us consider the value of the inner integral in (6.8),

$$
K(\mu)=\frac{1}{\sqrt{8 \pi} i} \int_{1-i \infty}^{1+i \infty} e^{P(\mu, s)} \frac{d s}{s}, \quad P(\mu, s)=\frac{(\mu+2 s)(\mu-s)^{2}}{6},
$$

for fixed $\mu$; this is the quantity that yields $K$ when integrated over the range $-\infty<\mu<\infty$. It is plotted for $-3 \leq \mu \leq 3$ in Figure 4 . 
We can argue, as we did following Theorem 5 in Section 10 , that $K(\mu)$ is exponentially small when $\mu \rightarrow-\infty$, and that $K(\mu)$ is of order $\mu^{-3 / 2}$ when $\mu \rightarrow+\infty$. Our strategy for evaluating $K$ will be to find a reasonable way to compute $K(\mu)$ when $|\mu|$ is small, together with a precise asymptotic estimate of $K(\mu)$ when $\mu$ is large.

First let's assume that $\mu$ is near zero. We have, by definition,

$$
K(\mu)=\frac{1}{\sqrt{8 \pi} i} e^{\mu^{3} / 6} I\left(\frac{\mu}{2}\right), \quad I(x)=\int_{1-i \infty}^{1+i \infty} e^{-x s^{2}+s^{3} / 3} \frac{d s}{s} .
$$

We can argue as in (A.6) that the path of integration can be bent so that $s$ begins at $\infty e^{-i \pi / 3}$ and ends at $\infty e^{+i \pi / 3}$; indeed, we may assume that $s$ traverses the path

$$
s=t+t^{-1}+\sqrt{3} i\left(t-t^{-1}\right) \quad \text { for } 0<t<\infty .
$$

Then the quantity $u=s^{3} / 3$ describes the contour

$$
u=-\frac{8}{3} t^{3}+4 t+4 \sqrt{3} i\left(t-t^{-1}\right)+4 t^{-1}-\frac{8}{3} t^{-3},
$$

which starts at $-\infty$ just below the negative real axis, stays below that axis and circles the origin counterclockwise, then returns (just above the axis) to $-\infty$. This is a contour $\Gamma$ for which we have Hankel's well-known formula

$$
\frac{1}{\Gamma(s)}=\frac{1}{2 \pi i} \int_{\Gamma} \frac{e^{u} d u}{u^{s}}
$$

Hence we can use the substitution $s=3^{1 / 3} u^{1 / 3}$ to write

$$
\begin{aligned}
I(x)=\frac{1}{3} \int_{\Gamma} e^{u} \exp \left(-3^{2 / 3} x u^{2 / 3}\right) \frac{d u}{u} & =\frac{1}{3} \int_{\Gamma} e^{u} \sum_{k \geq 0} \frac{\left(-3^{2 / 3} x\right)^{k} u^{2 k / 3-1}}{k !} d u \\
& =\frac{2 \pi i}{3} \sum_{k \geq 0} \frac{\left(-3^{2 / 3} x\right)^{k}}{k ! \Gamma(1-2 k / 3)} .
\end{aligned}
$$

(The sums are absolutely convergent.) This is the desired formula by which we can compute $K(\mu)$ when $|\mu|$ isn't too large. It can be expressed hypergeometrically in the form

$$
I(x)=\frac{2 \pi i}{3}\left(1-\frac{3^{2 / 3} x}{\Gamma\left(\frac{1}{3}\right)} F\left(\frac{5}{6}, \frac{1}{3} ; \frac{4}{3}, \frac{2}{3} ;-\frac{4}{3} x^{3}\right)-\frac{3^{1 / 3} x^{2}}{2 \Gamma\left(\frac{2}{3}\right)} F\left(\frac{7}{6}, \frac{2}{3} ; \frac{4}{3}, \frac{5}{3} ;-\frac{4}{3} x^{3}\right)\right) .
$$

Incidentally, it's interesting to apply this same idea to the integral (4.19). We obtain a formula that looks rather different from (A.13), namely

$$
s_{l, n}\left(e^{-\mu \nu}\right) \sim e^{\mu^{3 / 6}} \sqrt{\frac{\pi}{2}}\left(\frac{3^{-2 / 3}}{\Gamma\left(\frac{2}{3}\right)} F\left(\frac{1}{6} ; \frac{1}{3} ;-\frac{1}{6} \mu^{3}\right)-\frac{3^{-1 / 3}}{4 \Gamma\left(\frac{1}{3}\right)} F\left(\frac{5}{6} ; \frac{5}{3} ;-\frac{1}{6} \mu^{3}\right)\right) n^{1 / 6} .
$$


The quantity in parentheses is a confluent hypergeometric function,

$$
\frac{U\left(\frac{1}{6}, \frac{1}{3},-\frac{1}{6} \mu^{3}\right)}{2^{2 / 3} 3^{1 / 3} \pi^{3 / 2}}
$$

equating ( $A .19)$ with ( $A .13$ ) yields a known identity between Airy functions and confluent hypergeometrics [AS64, Equations 13.1.29 and 13.6.25]. We can also prove equality between individual "halves" of $(A .11)$ and $(A .19)$, using the hypergeometric identity

$$
e^{-z / 2} F(a ; 2 a ; z)=F\left(; a+\frac{1}{2} ; \frac{1}{16} z^{2}\right) \text {. }
$$

Now let's consider $K(\mu)$ as $\mu \rightarrow \infty$. Our experience with the similar integral (10.13) suggests that we try integrating along the path $s=\mu+i y / \sqrt{\mu}$. The integral reduces to

$$
K(\mu)=\frac{1}{\sqrt{8 \pi} \mu^{3 / 2}} \int_{-\infty}^{\infty} \frac{e^{-y^{2} / 2} \exp \left(-i y^{3} /\left(3 \mu^{3 / 2}\right)\right) d y}{1+i y / \mu^{3 / 2}}
$$

and we can obtain an asymptotic formula by expanding the real part of the integrand as $e^{-y^{2} / 2}$ times a power series in $y^{2}$ and $1 / \mu^{3}$. Namely, if we set $v=\mu^{-3 / 2}$ for convenience, we have

$$
\begin{aligned}
\frac{1}{2}\left(\frac{\exp \left(-i v y^{3} / 3\right)}{1+i v y}+\frac{\exp \left(+i v y^{3} / 3\right)}{1-i v y}\right)=1 & -\frac{18 y^{2}+6 y^{4}+y^{6}}{18} v^{2} \\
& +\frac{1944 y^{4}+648 y^{6}+108 y^{8}+12 y^{10}+y^{12}}{1944} v^{4} \\
& -\frac{524880 y^{6}+\cdots+y^{18}}{524880} v^{6}+\cdots
\end{aligned}
$$

Placing this inside $(A .21)$ and applying (4.14) gives

$$
K(\mu) \sim \frac{1}{2 \mu^{3 / 2}}\left(1-\frac{c_{1}}{\mu^{3}}+\frac{c_{2}}{\mu^{6}}-\frac{c_{3}}{\mu^{9}}+\cdots\right), \quad \text { as } \mu \rightarrow \infty,
$$

where

and so on.

$$
\begin{aligned}
& c_{1}=\frac{(1) 18+(1 \cdot 3) 6+(1 \cdot 3 \cdot 5) 1}{18}=\frac{17}{6} \\
& c_{2}=\frac{(1 \cdot 3) 1944+(1 \cdot 3 \cdot 5) 648+\cdots+(1 \cdot 3 \cdot 5 \cdot 7 \cdot 9 \cdot 11) 1}{1944}=\frac{1801}{72}
\end{aligned}
$$

However, we need to justify this expansion carefully because $\sum_{k \geq 0}(-1)^{k} c_{k} / \mu^{3 k}$ is divergent. The key is to show that $(A .23)$ is a strictly enveloping series, in the sense that its partial sums alternately overshoot and undershoot the true value of $K(\mu)$. The 
enveloping property is not difficult to prove, because we can show that series $(A .22)$ is enveloping with respect to $v^{2}$. If we remove all terms on the right side of $(A .22)$ that have degree greater than $2 k$ in $v$, the resulting sum is an upper bound or a lower bound for the function on the left side, according as $k$ is even or odd, for all real values of $v$ and $y$. This property is not difficult to prove, because the left side is

$$
\frac{\cos \left(v y^{3} / 3\right)-v y \sin \left(v y^{3} / 3\right)}{1+v^{2} y^{2}}
$$

and because the power series for cosine, sine, and $\left(1+v^{2} y^{2}\right)^{-1}$ are strictly enveloping [PS25, Problem I.142].

Incidentally, one can readily verify that the coefficient $c_{n}$ of $(A .23)$ can be expressed as a rather simple sum,

$$
c_{n}=\sum_{k=0}^{2 n} \frac{(2 n+2 k) !}{2^{n+k} 3^{k}(n+k) ! k !},
$$

because each term $y^{i} v^{j}$ of (A.22) arises from precisely one term in the expansion of (A.24). The denominator of $c_{n}$ turns out to be exactly

$$
2^{2 n-\nu_{2}(n)} 3^{\left(3 n-\nu_{3}(n)\right) / 2},
$$

where $\nu_{r}(n)$ denotes the sum of the digits of $n$ in radix-r notation.

Since the series $(A .23)$ for $K(\mu)$ is enveloping, we can integrate it term by term to get an enveloping series for the tail of the integral,

$$
\int_{\mu}^{\infty} K(\mu) d \mu \sim \frac{1}{\mu^{1 / 2}}\left(1-\frac{c_{1}}{7 \mu^{3}}+\frac{c_{2}}{13 \mu^{6}}-\frac{c_{3}}{19 \mu^{9}}+\cdots\right) .
$$

For any fixed $\mu$ this series is divergent, but we can find a "best" place to stop it (where the terms begin to increase in magnitude). For example, when $\mu=5$, the sum of the terms involving $c_{k}$ for $k \leq 21$ on the right of $(A .27)$ is 0.4458165587745 ; and the partial sum for $k \leq 22$ is 0.4458165587784 . So we know that

$$
0.4458165587745<\int_{5}^{\infty} K(\mu) d \mu<0.4458165587784
$$

These are the best lower and upper bounds attainable from $(A .27)$, because the next two partial sums are 0.4458165587744 and 0.4458165587787 . We obtain better accuracy as $\mu$ grows, and we get almost no information when $\mu$ is too small. For example, when $\mu=2$, the enveloping series $(A .27)$ tells us only that $.671<\int_{2}^{\infty} K(\mu) d \mu<.693$.

The integral of $K(\mu)$ from $-\infty$ to -4 is less than $10^{-5}$. A numerical integration over the range $-4 \leq \mu \leq 5$, using enough terms of the convergent series $(A .17)$ to ensure 
sufficient accuracy, now suffices to establish the value $K \approx 2.0337$, correct to four decimal places, as claimed in the introduction to this paper. (Such calculations are not quite trivial, because there is great cancellation between terms of $(A .17)$; according to $(A .15)$, the value of $I(\mu / 2)$ must be extremely small when $\mu$ is 3 or more, because $I(\mu / 2)$ must be multiplied by $e^{\mu^{3} / 6}$. The arithmetic leading to the stated result was done as far as possible with rational numbers; then high-precision values of $3^{2 / 3} / \Gamma\left(\frac{1}{3}\right)$ and $3^{1 / 3} / \Gamma\left(\frac{2}{3}\right)$ were used to combine the results.)

Substantially faster methods would need to be devised if we wanted to calculate $K$ to, say, 100 decimal places.

Acknowledgements. It is a pleasure to acknowledge here the help received in early stages of this work from several participants of the Random Graph ' 85 conference organized by M. Karoński in Poznań: J W. Moon made useful observations concerning Section 2; S. Janson obtained the limiting distribution (5.2) of cycle lengths, thereby greatly helping to guide some of the initial calculations; discussions with $\mathrm{H}$. Prodinger and $\mathrm{P}$. Kischenhoffer led to important clarifications of several points. 


\section{Bibliography}

[A.S64] Milton Abramowitz and Irene A. Stegun, Handbook of Mathematical Functions (Washington: National Bureau of Standards, 1964).

[Bo85] Béla Bollobás, Random Graphs (London: Academic Press, 1985).

[Bo89] Béla Bollobás, "Concentration of measure phenomena in the theory of random graphs," in Annals of Discrete Mathematics, Proceedings of Random Graphs '87, to appear.

[Br58] N. G. de Bruijn, Asymptotic Methods in Analysis (Amsterdam: North-Holland, 1958).

[CO70] Louis Comtet, Analyse Combinatoire, Tomes I et II (Paris: Presses Universitaires de France, 1970). English translation, Advanced Combinatorics (Dordrecht: D. Reidel, 1974).

[ER59] P. Erdős and A. Rényi, "On random graphs, I," Publ. Math. Debrecen 6 (1959), 290-297. Reprinted in Paul Erdös: The Art of Counting (MIT Press, 1973), 561-568.

[ER60] P. Erdős and A. Rényi, "On the evolution of random graphs," Magyar Tud. Akad. Mat. Kut. Int. Közl. 5 (1960), 17-61. Reprinted in Paul Erdös: The Art of Counting (MIT Press, 1973), 574-618.

[GJ83] Ian P. Goulden and David M. Jackson, Combinatorial Enumeration (New York: Wiley-Interscience, 1983).

[HP73] Frank Harary and Edgar M. Palmer, Graphical Enumeration (New York: Academic Press, 1973).

[Ja87] Svante Janson, "Poisson convergence and Poisson processes with applications to random graphs," Stochastic Processes and their Applications 26 (1987), 1-30.

[KS78] Donald E. Knuth and Arnold Schönhage, "The expected linearity of a simple equivalence algorithm," Theoretical Computer Science 6 (1978), 281-315.

[Pa85] Edgar M. Palmer, Graphical Evolution (New York: Wiley, 1985).

[Pi88] Boris Pittel, "On a random graph with a subcritical number of edges," Transactions of the American Mathematical Society 308 (1988), to appear.

[PS25] George Pólya and Gabor Szegö, Aufgaben und Lehrsätze aus der Analysis (Berlin: Springer, 1925). English edition, Problems and Theorems in Analysis (New York: Springer-Verlag, 1972).

[Wr77] E. M. Wright, "The number of connected sparsely edged graphs," Journal of Graph Theory 1 (1977), 317-330. 
